\title{
Nuclear Medium Modifications of Hadrons from Generalized Parton Distributions.
}

\author{
S. Liuti ${ }^{1, \text { 团 }}$ and S. K. Taneja ${ }^{1, \text { 团 }}$ \\ ${ }^{1}$ University of Virginia, Charlottesville, Virginia 22901, USA.
}

\begin{abstract}
We study the structure of generalized parton distributions in spin 0 nuclei within a microscopic approach for nuclear dynamics. GPDs can be used on one side as tools to unravel the deep inelastic transverse structure of nuclei in terms of both transverse spatial and transverse momentum degrees of freedom. On the other, one can obtain information on GPDs themselves by observing how they become modified in the nuclear environment. We derive the structure of the nuclear deeply virtual Compton scattering tensor and generalized parton distributions at leading order in $Q$ in a fieldtheoretical framework. The nuclear generalized parton distributions are calculated using a two step process - the convolution approach - where the scattering process happens from a quark inside a nucleon, itself inside a nucleus, disregarding final state interactions with both the nuclear and nucleon debris. We point out that details of the nuclear long range interactions such as twobody currents, can be disregarded compared to the deep inelastic induced modifications of the bound GPDs. We show how the pattern of nuclear modifications predicted, and in particular the deviations of off-shell effects from the longitudinal convolution provide clear signals to be sought in experimental measurements. Finally, we find interesting relationships by studying Mellin moments in nuclei: in particular we predict the $A$-dependence for the $D$-term of GPDs within a microscopic approach, and the behavior with $t$ of the total momentum carried by quarks in a nucleus. The latter provides an important element for the evaluation of nuclear hadronization phenomena which are vital for interpreting current and future data at RHIC, HERMES and Jefferson Lab.
\end{abstract}

PACS numbers: 13.60.-r, 12.38.-t, 24.85.+p

*s14y@virginia.edu

skt6c@virginia.edu 


\section{INTRODUCTION}

Generalized Parton Distributions (GPDs) have sensibly transformed our views and approaches on probing hadronic structure, since they were first introduced [1, 2, 3]. They provide, in fact, a framework to describe in a partonic language the orbital angular momentum carried by the nucleon's constituents [2]. In addition, they give invaluable information on the partonic distributions in the transverse direction with respect to the large longitudinal momentum in the reaction [4].

Recent developments focused on the relationship between GPDs, the Impact Parameter dependent Parton Distributions (IPPDs), and transversity [4, 5]. Several usually unaccounted for spatial observables including the transverse location of partons carrying a longitudinal fraction of momentum $x$, the interparton separation, and the radius of the proton, have been expressed in terms of both IPPDs and GPDs. The intrinsic transverse momentum distributions (or the Unintegrated Parton Distributions, UPDs), and their relation to the IPPDs, and GPDs, as well as to the nucleon Form Factors (FFs), and to the inclusive structure functions, can also be studied within the same context [5, 6, 7, 8]. A new perspective on GPDs as "tools" to study transversity in hadronic systems has just now begun to unravel 9].

The most straightforward method to access GPDs experimentally is to use a class of exclusive ep hard scattering experiments that proceed through the exchange of a highly virtual photon, the final state being the proton, or a proton resonance, and a real photon, or a vector meson. Initial experimental results exist to date for Deeply Virtual Compton Scattering (DVCS) off a proton target, a process that has also become the prototype for describing different aspects of GPDs (see e.g. Ref.10 for a review). Nevertheless, it is also clear that by using only this procedure, the mapping of the GPDs in a significant fraction of the phase space, and for a range of values of the process' scale might be quite lengthy.

GPDs were recently also measured through DVCS off nuclear targets [11]. The study of nuclear targets is particularly important as they provide a laboratory where additional information can be obtained on these elusive observables. Exploratory studies and estimates concentrating on the passage of nucleons through the nuclear medium, possibly generating Color Transparency, were performed in [6, 12, 13]. Another equivalently interesting possibility is that by keeping track of possible transverse deformations of nucleons in the nuclear medium, one can test the behavior of the GPDs themselves. In this paper we present a first investigation of this idea using a microscopic description of the nucleus.

The most clearcut experimental evidence that nucleons have a different deep inelastic structure inside the nuclear medium than when isolated, is represented by the EMC effect 14]. The EMC effect is the observation of a different behavior of the inclusive electron/muon nuclear cross section per nucleon with respect to the free nucleon one (see [15] for a review). These differences appear at intermediate values of Bjorken $x\left(x_{B j}=Q^{2} / 2 M_{N} \nu, Q^{2}\right.$ being the four-momentum transfer, $M_{N}$ the nucleon mass, $\nu$ the energy transfer), namely, $0.1 \lesssim$ $x_{B j} \lesssim 0.7$, where they cannot be attributed either to expected coherent effects such as nuclear shadowing $\left(x_{B j}<<0.1\right)$, or to Fermi motion $\left(x_{B j} \rightarrow 1\right)$. Throughout the years since the first discovery of the EMC effect an increasingly coherent picture has emerged confirming that even in a Deep Inelastic Scattering (DIS) process characterized by a high locality of the probe-target interaction region, nucleons cannot be treated as free. Their interactions

are instead important, they can bear as important consequences as being responsible for modifications of e.g. the chiral condensate, the color string tension, or the confinement scale 
in cold nuclear matter [16], ${ }^{1}$ and, therefore, play a key role in understanding the origin of hadronic mass.

Despite a quite general consensus on this picture, the form these interactions can take is still very model dependent, ranging from new applications of effective theories [19, 20] to extensive studies of the impact of modifications of the confinement scale, or of the string tension for bound nucleons ([16] and references therein). The extension to finite temperature measurements is possible but challenging, however at the light of current and future relativistic heavy ion collisions, and $e A$ collisions it is important to better define the connection with nuclear medium modifications that can be considered as precursors to the finite $T$ ones. The avenue that we espouse in this paper, is in this spirit. In particular, we make use of GPDs as a tool to access transverse coordinates and momenta in the nucleus. Transverse degrees of freedom, in turn, play a special role in quark re-interactions 21 and we expect them to play a significant role in nuclear medium modifications, and in hadronization [16]. In our description of inclusive DIS from a nucleus, re-interactions are parametrized as off-shell effects. Kinematical off-shell effects in a nucleus derive from the modification of the relation between the struck quark's transverse momentum, $k_{\perp}$, and its virtuality, $k^{2}$, produced by the $P^{2}$ dependence of the bound nucleon's structure function. It is well known that the account of kinematical off-shell effects alone is an oversimplified picture that produces a violation of Adler's sum rule 22, 23], and that dynamical nucleon off-shell effects should also be introduced. An important aspect of the approach proposed here is that, by restoring Adler's sum rule in a nucleus, it provides a description of the EMC effect that, at variance with the naive (on-mass-shell) binding models, can simultaneously, and quantitatively, reproduce both the $x_{B j}$ and $A$ dependences of the data.

In Section \we define our general framework; in Section ஹwe calculate GPDs in nuclei with offshell parametrizations. We subsequently evaluate in Section IV the role off-shellness in the second Mellin moments of the nuclear GPDs. Finally, in Section $\nabla$ we give our conclusions and outlook on future developments.

\section{GENERAL FRAMEWORK}

In this Section we present the formalism used to derive GPDs in nuclei in terms of their constituents, the protons and neutrons, including the effects of nucleon binding. Nuclear GPDs were at first calculated for the deuteron [26]. In Ref. 27] the general structure of the nuclear hadronic tensor within OPE was carefully evaluated for spin $0,1 / 2$, and 1 nuclei, however, a rather crude estimate of dynamical effects was given. The latter were evaluated in [28, 29] only in the low $x$ region, dominated by nuclear shadowing. Quantitative calculations for the valence region were performed in [30] for ${ }^{3} \mathrm{He}$, within the (longitudinal) convolution model, i.e. disregarding off-shell effects, and transverse degrees of freedom.

The results we present here are also valid in the valence region $(x \geq 0.2)$. Our evaluation of nuclear effects is extended to larger nuclei than the deuteron and ${ }^{3} \mathrm{He}$, namely to complex nuclei such as ${ }^{4} \mathrm{He},{ }^{12} \mathrm{C}$, etc..., that are close in atomic number to $\mathrm{Ne}$ and $\mathrm{Xe}$, used in HERMES experiments [1]]. Furthermore, we include nucleon off-shell effects. These play an essential role in the determination of nucleon deformations in the nuclear medium.

\footnotetext{
${ }^{1}$ Interactions become more and more important at finite temperature where non-perturbative properties of QCD, i.e. its vacuum, and confinement, are expected to be modified (see e.g. 18]).
} 
We consider DVCS off nuclei at leading order in $Q^{2}$ (twist-2). The diagrams in Fig 1 represent the two possible mechanisms through which DVCS off nuclei occurs: (a) coherent scattering, where the virtual photon with four-momentum $q$ scatters from a nuclear target producing a real photon $q^{\prime}$, the final nucleus $A$ recoiling as a whole; (b) incoherent scattering, where the final nucleus breaks up into a nucleon and an $A-1$ system, with final invariant mass squared, $M_{A}^{* 2} \approx M_{A}^{2}+$ "soft excitation terms". A similar treatment applies to the other hard exclusive reaction used as a probe of GPDs in nuclei, namely hard meson production. In what follows we calculate the "double layer" diagram (Fig 1a) because of its relation to the forward EMC effect. Current experimental data 11] contain in principle both contributions, a separation of the two being however feasible in the near future. In the present paper we are concerned with the coherent contribution only, because of its relation to the (forward) EMC effect. A comparison of the contributions of coherent and incoherent scattering will be carried out in [37]. Other components besides the nucleon ( $\Delta$ 's, 6-quark bags, etc...) could in principle be similarly described by the lower part of the diagram. In this paper we take the point of view that both the $x_{B j}$ and $A$ dependences of the EMC effect can be explained quantitatively within a "minimal" approach involving only nucleons. The nucleon's offshellness however has to be considered explicitly, as it plays the role of a parametrization of soft interactions among nucleons (or quarks and nucleons debris) during the scattering process.

We denote by $P_{A}, P, k$, the nuclear, the active nucleon's and active quark's four-momenta, respectively. $P_{A}^{\prime}=P_{A}-\Delta, P^{\prime}=P-\Delta$, and $k^{\prime}=k-\Delta$ are the final nuclear, nucleon's and quark's momenta, respectively; $q$ is the virtual photon momentum, and $q^{\prime}=q+\Delta$, the outgoing photon momentum; $\Delta^{2} \equiv t$. Notice that $\Delta=k-k^{\prime} \equiv P-P^{\prime} \equiv P_{A}-P_{A}^{\prime}$, in the absence of final state interactions.

Two distinct formulations of GPDs are given in the literature, that differ in the "choice of the defining four-vector" 34]. Since both formulations turn out to be useful in a complementary way, in the following we list the kinematical variables and definitions for both.

In set $(\mathbf{1})$ the kinematical variables are defined with respect to the average of the target's incoming and outgoing momenta following [2]. These are: $\bar{P}_{A}=\left(P_{A}+P_{A}^{\prime}\right) / 2$, and $\bar{P}=$ $\left(P+P^{\prime}\right) / 2$, for the nucleus and for the off-shell $\left(P^{2} \neq M^{2}\right)$ nucleon, respectively. One has : 2

$$
\begin{aligned}
x & =\left(k^{+}+k^{+}\right) / 2\left(\bar{P}_{A}^{+} / A\right) \\
y & =\bar{P}^{+} /\left(\bar{P}_{A}^{+} / A\right) \\
x_{N} & =x / y \equiv\left(k^{+}+k^{\prime+}\right) / 2 \bar{P}^{+} \\
\xi & =\Delta^{+} / 2\left(\bar{P}_{A}^{+} / A\right) \\
\xi_{N} & =\Delta^{+} / 2 \bar{P}^{+} \equiv \xi / y
\end{aligned}
$$

The resulting distributions are real, and they are even functions of the variables $\xi, \xi_{N}$.

If the variables are defined with respect to the incoming nucleon momentum as in [3] one

${ }^{2}$ We use the notation: $p^{ \pm}=\frac{1}{\sqrt{2}}\left(p_{o} \pm p_{3}\right)$, with $(p k)=p^{+} k^{-}+p^{-} k^{+}-p_{\perp} \cdot k_{\perp}$. 
has:

$$
\begin{aligned}
X & =k^{+} /\left(P_{A}^{+} / A\right) \\
Y & =P^{+} /\left(P_{A}^{+} / A\right) \\
X_{N} & =X / Y \equiv k^{+} / P^{+} \\
\zeta & =\Delta^{+} /\left(P_{A}^{+} / A\right) \\
\zeta_{N} & =\Delta^{+} / P^{+} \equiv \zeta / Y
\end{aligned}
$$

The structure functions defined in terms of these variables are more straightforwardly connected with the DIS parton distributions.

GPDs written in terms of the two sets of variables can be translated into one another. The graph in Fig 1 in fact, simultaneously defines three configurations where: i) both $k$ and $k^{\prime}$ are quarks; ii) both $k$ and $k^{\prime}$ are antiquarks; iii) $k$ is a quark (antiquark) and $k^{\prime}$ is an antiquark (quark).

In the quark region, that is the main interest for this paper because it is where the EMC effect in DIS occurs, $\xi<x<1, \zeta<X<1$, and the following relations hold:

$$
\begin{aligned}
X & =\frac{x+A \xi}{1+\xi} \\
Y & =\frac{y+A \xi}{1+\xi} \\
X_{N} & =\frac{x+\xi}{y+\xi} \\
\zeta & =\frac{2 \xi}{1+\xi / A} \\
\zeta_{N} & =\frac{2 \xi_{N}}{1+\xi_{N}}
\end{aligned}
$$

Similar relations can be derived in the antiquark sector $(-1<x<-\xi$ and $\zeta<X<1){ }^{3}$

\section{A. Nucleon}

We first summarize the main results in the free nucleon case. The amplitude for Deeply Virtual Compton Scattering (DVCS) is defined as:

$$
T^{\mu \nu}(P, \Delta, q)=i \int d^{4} y e^{i q \cdot y}\left\langle P^{\prime}\left|T\left(J^{\mu}(y) J^{\nu}(0)\right)\right| P\right\rangle .
$$

Notice that we will only assume, and not write explicitly, the dependence of the various terms on the virtual photon's momentum, $q$. The factorization theorem for hard scattering processes (Fig (1) allows one to separate the hard part, calculated using QCD Feynmann rules, from the soft hadronic matrix element, $\mathcal{M}:{ }^{4}$

$$
T^{\mu \nu}=-i \int \frac{d^{4} k}{(2 \pi)^{4}} \operatorname{Tr}\left[\left(\frac{\gamma^{\nu} i(\not k+\not q) \gamma^{\mu}}{(k+q)^{2}+i \epsilon}+\frac{\gamma^{\mu} i(\not k+\not \Delta-\not q) \gamma^{\nu}}{(k+\Delta-q)^{2}+i \epsilon}\right) \mathcal{M}(k, P, \Delta)\right] .
$$

\footnotetext{
${ }^{3}$ The region $-\xi<x<\xi$ requires a more detailed description and it is beyond the scope of this paper. See however 34 for the nucleon case.

${ }^{4}$ We do not include for the time being either radiative corrections, or $\mathcal{O}\left(1 / Q^{2}\right)$ terms.
} 
$\mathcal{M}(k, P, \Delta)$ is an off-forward correlation function:

$$
\mathcal{M}_{i j}(k, P, \Delta)=\int d^{4} y e^{i k \cdot y}\left\langle P^{\prime}\left|\bar{\psi}_{j}(-y / 2) \psi_{i}(y / 2)\right| P\right\rangle,
$$

with Dirac indices $i, j$ written out explicitly. Notice that we have written the argument of $\psi$ choosing the "symmetrical" formalism of [2]. By taking the Bjorken limit, and by projecting out the dominant contribution corresponding to the transverse virtual photon polarization, one obtains:

$$
T^{\mu \nu}=\left(-g^{\mu \nu}+\frac{\widetilde{P}^{\mu} \widetilde{P}^{\nu}}{\widetilde{P}^{2}}-\frac{q^{\mu} q^{\nu}}{Q^{2}}\right) \mathcal{F}_{T}
$$

where $\widetilde{P}_{\mu}=\bar{P}_{\mu}+\left(\bar{P} \cdot q / Q^{2}\right) q_{\mu}$. The off-forward structure function can then be extracted from $\mathcal{F}_{T}$ as: ${ }^{5}$

$$
\mathcal{F}_{T}(P, \Delta)=\int_{-1}^{1} d x\left(\frac{1}{x-\xi / 2+i \epsilon}-\frac{1}{x+\xi / 2-i \epsilon}\right) F(x, \xi, t),
$$

with:

$$
F(x, \xi, t)=\frac{1}{2 \bar{P}^{+}}\left[\bar{U}\left(P^{\prime}, S^{\prime}\right)\left(\gamma^{+} H(x, \xi, t)+\frac{i \sigma^{+\mu} \Delta_{\mu}}{2 M} E(x, \xi, t)\right) U(P, S)\right]
$$

Eq. (91) defines the GPDs, $H$ and $E$, for the unpolarized scattering case [2]. $H$ and $E$, and the generalized distributions, $\mathcal{F}_{\zeta}(X, t)$ and $\mathcal{K}_{\zeta}(X, t)$ introduced similarly by Radyushkin using Double Distributions (DD) in [3] describe the same physics, however the connection between the two different sets of kinematical variables, and physical regimes spanned needs to be specified.

In this paper, we adopt the convention first illustrated in [34], that is we define $H_{q(\bar{q})}(X, \zeta, t)$, and $E_{q(\bar{q})}(X, \zeta, t),{ }^{6}$ directly in terms of $H$ and $E$, as from Eq. (99), but: i) Changing the kinematical variables to set (2), with $A=1$ for the free nucleon; ii) Defining explicitly the contributions of quarks and antiquarks. Because we are interested in the region dominated by DGLAP evolution (and $\xi>0$ ), we consider the following mapping of both regions: $\xi<x<1$ describing scattering from a quark, and $-1<x<-\xi$ describing scattering from an antiquark onto $\zeta<X<1$ :

$$
\begin{aligned}
(1-\zeta / 2) H_{q}(X, \zeta, t) & =H(x, \xi, t) \quad x>\xi, \quad X>\zeta \\
(1-\zeta / 2) H_{\bar{q}}(X, \zeta, t) & =-H(x, \xi, t) \quad x<-\xi, \quad X>\zeta
\end{aligned}
$$

and

$$
\begin{aligned}
& (1-\zeta / 2) E_{q}(X, \zeta, t)=E(x, \xi, t) \quad x>\xi, \quad X>\zeta \\
& (1-\zeta / 2) E_{\bar{q}}(X, \zeta, t)=-E(x, \xi, t) \quad x<-\xi, \quad X>\zeta,
\end{aligned}
$$

\footnotetext{
${ }^{5}$ Notice that we adopt the axial gauge, although results can be cast in a form highlighting gauge invariance [2].

${ }^{6}$ For completeness, the correspondence with the notaion in [34] is: $H_{q(\bar{q})} \equiv \mathcal{F}_{q(\bar{q})}(X, \zeta, t)$, and $E_{q(\bar{q})} \equiv$ $\mathcal{K}_{q(\bar{q})}(X, \zeta, t)$
} 
where the factor $1-\zeta / 2 \equiv \bar{P}^{+} / P^{+}$is due to the difference in "reference momenta" (see Eqs.(11). The new definitions are best suited both for describing the convolution with nuclear variables (see below), as well as for perturbative evolution. Notice that for $\xi, \zeta=0$, for instance, one maps the entire $X \in[0,1]$ domain onto the $x \in[-1,1]$ domain. As a result, $H_{q}(X, \zeta, t)$ and $H_{\bar{q}}(X, \zeta, t)$, can be expressed in terms of the Non-Singlet (NS), or Valence $(\mathrm{V})$, and Singlet $(\mathrm{S})$ contributions as:

$$
\begin{aligned}
H^{V} & =\sum_{q=u, d} H_{q}(X, \zeta, t)-H_{\bar{q}}(X, \zeta, t) \\
H^{S} & =\sum_{q=u, d, s, \ldots} H_{q}(X, \zeta, t)+H_{\bar{q}}(X, \zeta, t)
\end{aligned}
$$

$H_{V}$ and $H_{S}$ become the usual valence and singlet quark distributions in the forward limit.

The proton and neutron GPDs are obtained from $H_{q(\bar{q})}$ as:

$$
\begin{aligned}
H^{p} & =\frac{2}{3} H_{u}-\frac{1}{3} H_{d}-\frac{1}{3} H_{s} \\
H^{n} & =-\frac{1}{3} H_{u}+\frac{2}{3} H_{d}-\frac{1}{3} H_{s} .
\end{aligned}
$$

Similar formulae hold for $E_{q(\bar{q})}$. In what follows we assume that isospin invariance holds in a nucleus as well.

In Section III we calculate GPDs using an effective theory for the proton, with a quark and a diquark component, as the lowest light-cone Fock state wavefunction.

\section{B. Nucleus}

We consider now the case in which the nucleon is bound in a nucleus with mass number $A$ and mass $M_{A}$. The off-forward nuclear amplitude can be calculated analogously to Eq. (15) by convoluting the truncated off-forward nucleon amplitude, $T_{\mu \nu}^{N}$, with the nuclear matrix element, $\mathcal{M}^{A}\left(P, P_{A}, \Delta\right)$ :

$$
T_{\mu \nu}^{A}\left(P_{A}, \Delta\right)=\int \frac{d^{4} P}{(2 \pi)^{4}} T_{\mu \nu}^{N}(k, P, \Delta) \mathcal{M}^{A}\left(P, P_{A}, \Delta\right),
$$

where:

$$
\mathcal{M}_{i j}^{A}\left(P, P_{A}, \Delta\right)=\int d^{4} y e^{i P \cdot y}\left\langle P_{A}^{\prime}\left|\bar{\Psi}_{A, j}(-y / 2) \Psi_{A, i}(y / 2)\right| P_{A}\right\rangle .
$$

$T_{\mu \nu}^{N}(k, P, \Delta)$ is the hadronic tensor for a bound nucleon (we consider from now on the isoscalar combination, $N=(p+n) / 2)$.

The structure function can be extracted from Eq.(14) similarly to the procedure used to obtain Eq.(91) from Eq.(15), leading to:

$$
F^{A}(X, \zeta, t)=\int \frac{d^{4} P}{(2 \pi)^{4}} F_{O F F}^{N}\left(X_{N}, \zeta_{N}, P^{2}, t\right) \mathcal{M}^{A}\left(P, P_{A}, \Delta\right)
$$

where all kinematical variables have been defined in Eqs.1123). Notice that the bound nucleon is off its mass shell, namely: $P^{2} \equiv P^{2}\left(X, P_{T}^{2}\right) \neq M^{2}$, and consequently 
$F_{O F F}^{N}\left(X_{N}, \zeta_{N}, P^{2}, t\right) \neq F^{N}\left(X_{N}, \zeta_{N}, t\right) . \quad F^{N}\left(X_{N}, \zeta_{N}, t\right)=\left(F^{p}+F^{n}\right) / 2$ is the nucleon, or isoscalar, combination of GPDs, obtained using Eqs.(13b).

The form of $\mathcal{M}_{A}$ depends on the spin of the nucleus. In the next Section we evaluate Eq.(16) for a nucleus with spin 0 .

\section{EVALUATION OF NUCLEAR GPDS}

The evaluation of Eq.(16) requires a mechanism for describing nucleon off-shellness. We adopt an effective theory described in Fig 2, where the coupling at the nucleon vertex is: $g\left(k^{2}\right) \bar{\psi}_{N} \psi_{q} \phi, \psi_{N}, \psi_{q}$ and $\phi$ being the wave functions of the nucleon, $P$, the active quark, $k$, and the spectator diquark, $k_{X}$, respectively. Within this model, that can be considered a realization of the lowest Fock component for the light cone wave function, the active quark's virtuality, $k_{\mu}^{2} \neq m_{q}^{2}$, is related to the intrinsic transverse momentum, and either variable can be integrated over to obtain the forward parton distribution (see e.g. [31] and references therein). Similarly, one can treat the "active nucleon" within a relativistic nuclear effective theory (lower part of diagram in Fig 2). Different versions of the spectator model were used to calculate the (forward) EMC effect [23, 25, 35].

The main conclusion from these papers was that nucleon off-shell effects are essential for reproducing quantitatively the effect although the dynamical source of off-shellness was not transparent. In Fig [3 we present a calculation of the ratio $R_{A}^{D I S}=d \sigma_{A} / d \sigma_{D}$ obtained including off-shell effects.

In order to interpret the curves in the figure, a few comments are in order:

i) For consistency with the presentation of experimental data, we show ratios of forward nuclear structure functions (with $A>2$ ), to the deuteron forward structure function. The deuteron is in itself affected by an EMC effect [35]. Ratios of $A>2$ nuclei to a nucleon structure function will in principle look different.

ii) The dashed curve is calculated according to the longitudinal convolution, i.e. with no offshell effect included. The effect of binding is enhanced with respect to mean field models [19] because of the account of short range correlations among nuclei 38]. We note however that despite the differences between the models in [19] and [38], neither one is able to reproduce the effect for the measured range of values of nuclei. In other words binding cannot account for the $A$ dependence of the effect.

iii) As already noted in [23, 25], kinematical off-shell effects, coming from the different relation between the active quark's virtuality and its transverse momentum in a nucleus, by generating an extra $A$-dependent term, can account for the discrepancies at large $x$. This is clearly seen from Fig 3 .

iv) Dynamical off-shell effects, originating from intrinsic modifications of the quark spectral function need to be taken into account in order to guarantee the fulfillment of Adler's Sum Rule [24] in nuclei. Forward DIS, besides allowing us to state that dynamical off-shell effects should indeed be present, gives very little insight on the nature of the effects. Our calculation presented in Fig [3, includes the effect of parton reinteractions, located mainly at low $x(x<0.3)$ [39]. This seems to be a favored mechanism with respect to the nucleon deformation one, taken into account in [23].

v) Most crucially, nucleon binding alone does not provide the correct $A$-dependence of the EMC effect ratio because of the saturation with in increasing $A$ of both the kinetic and separation energies of nucleons' [19, 42]. Off-shell effects provide an extra degree of freedom that allows for a quantitative description of the effect. 
GPDs represent a theoretical tool that can be used for studies of both parton reinteractions [40], and of nucleons spatial dimensions [4, 5, 6].

It is therefore natural to extend our approach to the off-forward case. We discuss the region: $\zeta \leq X$, which is most relevant for the EMC effect, where both partons in Fig 1 are quarks (an extension to the region $\zeta>X$, where GPDs behave like meson distributions, will be considered in a forthcoming paper).

\section{A. Nucleon}

In the spectator model for the nucleon [6], the outgoing diquark has spin/isospin either 0 or 1 . Its mass is in principle described by a mass distribution or spectral function. In our calculations we consider only the scalar component of the diquark. Furthermore, we consider the mass spectrum to be dominated by a single mass value. Both assumptions can in principle be lifted at a later stage. However, for our goal of studying nuclear effects, we do not expect such details of the model to produce significant changes. The structure of the nucleon matrix element can be read from the upper part of Fig 2

$$
\mathcal{M}_{i j}^{N}=\bar{U}\left(P^{\prime}, S\right) \bar{\Gamma}\left(k^{\prime}, P\right) \frac{\left(\not k^{\prime}+m\right)}{k^{\prime 2}-m^{2}} \frac{(\not k+m)}{k^{2}-m^{2}} \Gamma(k, P) U(P, S),
$$

where $\Gamma$ is the vertex connecting the quark, diquark, and nucleon; $U(P, S)$ is the nucleon spinor. The form of $\Gamma$ depends on the way the spin and isospin are carried through the vertex. In analogy with the forward case [25], we define $\Gamma$ so that: ${ }^{7}$

$$
\mathcal{M}_{i j}^{N}=\rho_{N}\left(k^{2}, k^{\prime 2}\right) \sum_{s} u_{i}(k, s) \bar{u}_{j}\left(k^{\prime}, s\right) 2 \pi \delta\left(k_{X}^{2}-M_{X}^{2}\right)
$$

with

$$
\rho_{N}\left(k^{2}, k^{\prime 2}\right)=\mathcal{N} \frac{\phi\left(k^{\prime 2}\right)}{k^{\prime 2}-m^{2}} \frac{\phi\left(k^{2}\right)}{k^{2}-m^{2}},
$$

$\mathcal{N}$ being a normalization constant. In Eq.(18) the active off-shell parton is described by a spinor that obeys the Dirac equation, with an off-shell mass, $m^{*}\left(m^{*}=\left(m+\sqrt{k^{2}}\right) / 2\right)$ :

$$
(m+\not k) u(k, s)=2 m^{*} u(k, s)
$$

By inserting Eqs.(17) and (18) in Eqs.(5) and (9) one obtains:

$$
\begin{gathered}
\sqrt{1-\zeta} H^{N}(X, \zeta, t)-\frac{1}{4} \frac{\zeta^{2}}{\sqrt{1-\zeta}} E^{N}(X, \zeta, t)= \\
=\frac{1}{2 P^{+}} \int \frac{d^{4} k}{(2 \pi)^{4}} \operatorname{Tr}\left[\gamma^{+} \mathcal{M}^{N}(k, P, \Delta)\right]= \\
=\frac{X}{1-X} \sqrt{\frac{X-\zeta}{X}} \int \frac{d^{2} k_{\perp}}{(2 \pi)^{3}} \rho_{N}\left(k^{2}, k^{\prime 2}\right) .
\end{gathered}
$$

\footnotetext{
${ }^{7}$ Similar results were obtained in 31] by assuming $\Gamma=\hat{I} \Phi\left(k^{2}, P^{2}\right)$. The complete set of independent functions that appear in principle using a covariant formalism for the off-shell tensor was listed in 32 ].
} 
The square root factor in the equation takes into account the normalization of the spinors from Eq.(20). In our derivation we made use of Eqs.(10); all kinematical variables have been defined according to Eqs.(2) with $A=1$. More details are given in the Appendix.

The results obtained above correctly reproduce the parton model in the forward limit where:

$$
\bar{U}(P, S) \bar{\Gamma}(k, P) \frac{(\not k+m)}{k^{\prime 2}-m^{2}} \frac{(\not k+m)}{k^{2}-m^{2}} \Gamma(k, P) U(P, S) \approx \rho_{N}\left(k^{2}\right)(\not k+m),
$$

yielding for the (transverse) structure function:

$$
\begin{aligned}
F^{N}\left(x_{B j}\right) & =\frac{1}{2 P^{+}} \int \frac{d^{4} k}{(2 \pi)^{4}} \rho_{N}\left(k^{2}\right) \operatorname{Tr}\left[\gamma^{+}(\not k+m)\right] \\
& =\frac{x_{B j}}{1-x_{B j}} \int \frac{d^{2} k_{\perp}}{(2 \pi)^{3}} \rho_{N}\left(x, k_{\perp}^{2}\right)
\end{aligned}
$$

with $\rho_{N}\left(k^{2}, k^{\prime 2}\right) \rightarrow \rho_{N}\left(k^{2}\right)$ for $k=k^{\prime}$.

\section{B. Nucleus}

The nuclear part of the diagram in Fig 2 is evaluated similarly to the nucleon one. For a spin 0 nucleus we write the matrix element, Eq.(14), as:

$$
\mathcal{M}_{i j}^{A}=\bar{U}_{A-1}\left(P_{A}^{\prime}, S\right) \bar{\Gamma}_{A} \frac{\left(P^{\prime}+M\right)}{P^{\prime 2}-M^{2}} \frac{(P+M)}{P^{2}-M^{2}} \Gamma_{A} U_{A-1}\left(P_{A}, S\right) 2 \pi \delta\left(P_{A-1}^{2}-M_{A-1}^{* 2}\right)
$$

where $U_{A-1}$ describes the $A-1$ system, and $\Gamma_{A}$ is the nuclear vertex function, and $M_{A-1}^{*}$ is the mass of the outgoing $A-1$ nuclear system.

We now make the assumption that the spin structure at the nuclear vertex is treated similarly to Eq.(18):

$$
\mathcal{M}_{i j}^{A}=\rho_{A}\left(P^{2}, P^{\prime 2}\right) \sum_{S} U_{i}(P, S) \bar{U}_{j}\left(P^{\prime}, S\right)
$$

$U_{i}(P, S)$ being the spinor for an off-shell nucleon with effective mass $M^{*} \neq M . \rho_{A}\left(P^{2}, P^{\prime 2}\right)$ is the off-diagonal nucleon light cone momentum distribution. It can be approximated by a non relativistic nuclear spectral function:

$$
\begin{aligned}
\rho_{A}\left(P^{2}, P^{\prime 2}\right) & \approx S_{A}\left(|\mathbf{P}|,\left|\mathbf{P}^{\prime}\right|, E\right) \\
& =\sum_{f} \Phi_{f}(|\mathbf{P}|) \Phi_{f}^{*}\left(\left|\mathbf{P}^{\prime}\right|\right) \delta\left(E-\left(E_{A-1}^{f}-E_{A}\right)\right),
\end{aligned}
$$

where $|\mathbf{P}|$, and $\left|\mathbf{P}^{\prime}\right|$ are the absolute values of the incoming and outgoing nucleons threemomenta, respectively, $E$ is the nucleon separation energy, $E_{A}$ being the binding energies of the initial nucleus, $A$, and of the final nuclear system, $A-1 . \Phi_{f}$ is the (Fourier transformed) overlap integral between the initial and final nuclear wave functions; the sum over $f$ is carried out over all the final configurations of the $A-1$ system (see [38] and references therein for more details). Eqs.(25) and (21), inserted in (Eq.(16)), yield:

$$
F^{A}(X, \zeta, t)=\int \frac{d^{2} P_{\perp} d Y}{2(2 \pi)^{3}} \frac{1}{(A-Y)} \mathcal{A} \rho_{A}\left(P^{2}, P^{\prime 2}\right) F_{O F F}^{N}\left(X_{N}, \zeta_{N}, P^{2}, t\right),
$$


where $\mathcal{A}=(Y-\zeta / 2)(\sqrt{Y(Y-\zeta)})$ accounts for the spinors normalization (Eq.(25) $)$. By replacing $F_{O F F}^{N}$ with the expression in Eq.(21), one finally obtains:

$$
\begin{array}{r}
H^{A}(X, \zeta, t)=\int \frac{d^{2} P_{\perp} d Y}{2(2 \pi)^{3}} \frac{1}{(A-Y)} \rho_{A}\left[P^{2}\left(Y, P_{\perp}^{2}\right), P^{\prime 2}\left(Y, P_{\perp}^{2}, \zeta, t\right)\right] \mathcal{A} \\
\times \sqrt{\frac{Y-\zeta}{Y}}\left[H_{O F F}^{N}\left(\frac{X}{Y}, \frac{\zeta}{Y}, P^{2}, t\right)-\frac{1}{4} \frac{(\zeta / Y)^{2}}{1-\zeta / Y} E_{O F F}^{N}\left(\frac{X}{Y}, \frac{\zeta}{Y}, P^{2}, t\right)\right]
\end{array}
$$

The kinematical variables are from Eqs.(2); similarly to the free nucleon case. Numerical results presented in the following where obtained in the small $\zeta$ approximation, i.e. considering for the off-shell nucleon:

$$
\sqrt{1-\zeta_{N}} H_{O F F}^{N}\left(X_{N}, \zeta_{N}, P^{2}, t\right)=\frac{X_{N}}{1-X_{N}} \sqrt{\frac{X_{N}-\zeta_{N}}{X_{N}}} \int \frac{d^{2} k_{\perp}}{2 \pi} \widetilde{\rho}_{N}\left[k^{2}\left(P^{2}\right), k^{\prime 2}\left(P^{2}\right)\right]
$$

and disregarding the second term in Eq.(29). $H_{O F F}^{N}$ is modified both kinematically and dynamically with respect to the free nucleon one, $H^{N}$ (Eq.(21) ). Kinematical modifications due to Fermi motion and nuclear binding produce an extra shift in the $X$ dependence with respect to the free nucleon one, analogous to what found in forward DIS. $H_{O F F}^{N}$ is however expected to be also structurally different from the on-shell case because of nuclear medium induced distortions (schematically, $\widetilde{\rho} \neq \rho$ ). Off-shell modifications, differently from Fermi motion and binding, affect the transverse variables. It is therefore of the out-most importance to evaluate them using GPDs, with a twofold goal in mind: On one side because of the interpretation of GPDs as Fourier transforms of Impact Parameter dependent Parton Distribution Functions (IPPDFs), such studies will provide a handle to directly evaluate the spatial modifications of the nucleon inside the nuclear medium [37]. The other compelling reason to explore quantitatively off-shell effects in this context is that they are essential in order to interpret the experimental data on DIS from nuclei (see Fig [3).

Kinematical off-shell effects can be evaluated straightforwardly as they result from a difference in the relationship between the struck parton's virtuality, $k_{\mu}^{2} \equiv k^{2}$, and its intrinsic transverse momentum, $k_{\perp}$ in a free and in a bound nucleon, respectively:

$$
\begin{aligned}
k_{N}^{2} & =\mathcal{M}^{2}(X)-\frac{\mathbf{k}_{\perp}^{2}}{1-X} \\
k_{A}^{2} & =\mathcal{M}_{A}^{2}\left(X_{N}\right)-\frac{\mathbf{a}_{\perp}^{2}}{1-X_{N}}
\end{aligned}
$$

with:

$$
\begin{aligned}
\mathcal{M}^{2}(X) & =X M^{2}+\frac{X}{1-X} M_{X}^{2} \\
\mathcal{M}_{A}^{2}\left(X_{N}\right) & =X_{N} P^{2}-\frac{X_{N}}{1-X_{N}} M_{X}^{2} \\
P^{2} & =\frac{Y}{A}\left(M_{A}^{2}-\frac{M_{A-1}^{2}+P_{\perp}^{2}}{1-Y / A}-\frac{P_{\perp}^{2}}{Y / A}\right) .
\end{aligned}
$$

where $X_{N}=X / Y, m_{q q}$ is the diquark's mass, and the intrinsic transverse momentum in the nucleus is: $\mathbf{k}_{\perp}-X_{N} \mathbf{p}_{\perp} \equiv \mathbf{a}_{\perp}$. 
In a nucleus one has therefore a shift in the longitudinal variable, $X \rightarrow X_{N}$, a shift in the transverse variable, $\mathbf{k}_{\perp} \rightarrow \mathbf{k}_{\perp}-X_{N} \mathbf{p}_{\perp}$, and a shift in the nucleon's invariant mass squared, $M^{2} \rightarrow P_{\mu}^{2}$. The shift in $X$ is associated with the $X$-rescaling caused by binding. The shifts in the transverse variables are due to off-shell effects. Even in the absence of nucleon deformations $(\widetilde{\rho}=\rho)$, these shifts modify the bound GPD, $H_{O F F}^{N}$ in Eq.(29) $)$. It is important to notice that such modification is based on the same physical mechanism as for the bound forward structure functions. By changing variables from $k_{\perp}$ to $k_{\mu}^{2}$, and by writing explicitly $\rho$ as in Eq. (19), one can in fact write:

$$
\begin{aligned}
H_{O F F}^{N}\left(X_{N}, \zeta, P^{2}, t\right) & =X \sqrt{\frac{X_{N}}{\zeta-X_{N}}} \int_{0}^{2 \pi} d \phi \int^{k_{A M A X}^{2}\left(X_{N}\right)} \frac{d k_{A}^{2}}{2 \pi} \frac{\phi\left(k_{A}^{2}\right) \phi^{*}\left(k_{A}^{\prime 2}\right)}{k_{A}^{2} k_{A}^{\prime 2}}, \\
H^{N}(X, \zeta, t) & =X \sqrt{\frac{X}{\zeta-X}} \int_{0}^{2 \pi} d \phi \int^{k_{N M A X}^{2}(X)} \frac{d k_{N}^{2}}{2 \pi} \frac{\phi\left(k_{N}^{2}\right) \phi^{*}\left(k_{N}^{\prime 2}\right)}{k_{N}^{2} k_{N}^{\prime 2}}
\end{aligned}
$$

The difference between $H_{O F F}^{N}$ and $H^{N}$ is in the upper limit of integration for the equations above. The effect of kinematical off-shellness can therefore be obtained as an additional rescaling of the longitudinal variable, $X$, that turns out to increase the effect of Fermi motion and binding. Phrased otherwise, kinematical off-shell effects are an indirect consequence of Fermi motion and binding that, although originating from modifications of transverse variables in a nucleus, affect the dependence of the GPDs on the longitudinal variable $X$.

The existence of kinematical off-shellness indirectly implies that intrinsic deformations/parton reinteractions are present. In other words, off-shell effects are an indirect manifestation of the impact of interactions among particles during the hard scattering process. A clear illustration of this picture was given, for example, in [22] where it was shown that in DIS from a nucleon kinematical off-shell effects can generate $F_{L}$; however, this can be evaluated consistently with baryon number conservation (Adler's sum rule [24]) only by including $a b$ initio interactions, in this case the higher twist terms. In the nuclear case a full theory of parton re-interactions is still lacking (see however many aspects treated e.g. in [40] and references therein), therefore in what follows we introduce a phenomenological approach. We first calculate exactly the kinematical off-shell effects. We then restore Adler Sum Rule by introducing interactions through a modification of spectral function's denominator, Eq.(19). A more detailed discussion of this point is beyond the scope of this paper and will be given elsewhere [39].

If instead off-shell effects are disregarded, one recovers a longitudinal "convolution formula":

$$
H^{A}(X, \zeta, t)=\int_{X}^{A} d Y \sqrt{\frac{Y-\zeta}{Y}} f_{A}(Y, \zeta, t) H^{N}\left(X_{N}, \zeta, t\right),
$$

where for consistency with previous literature in forward scattering [15], in Eq.(29) we switch integration variables from $P_{\perp}$ to $|P| \equiv P$ :

$$
f_{A}(Y, \zeta, t)=2 \pi M \int_{P_{\min }(Y, \bar{E})^{\infty}} d P P \Phi_{A}(P) \Phi_{A}^{*}(|\mathbf{P}+\boldsymbol{\Delta}|)
$$


Other kinematical variables that appear in the equation are:

$$
\begin{aligned}
|\mathbf{P}+\boldsymbol{\Delta}| & =\left(P^{2}+\zeta^{2} P_{\|}^{2}+\Delta_{\perp}^{2}+2 P_{\|} \zeta+2 P_{\perp} \cdot \Delta_{\perp}\right)^{2} \\
P_{\|} & \approx(M-\bar{E})-M Y \\
P_{\min }(Y, \bar{E}) & =|M(1-Y)-\bar{E}| .
\end{aligned}
$$

$\bar{E}=\int d^{3} P d E S_{A}(P, E) E$, is the average separation energy. Its value, enhanced by nucleon short range correlations, was shown to govern binding effects in the forward EMC effect (see [15] and references therein). By performing the integration over $|\mathbf{P}| \equiv P=\left(P_{\|}^{2}+P_{\perp}^{2}\right)^{1 / 2}$, instead than over the transverse momentum $P_{\perp}$, one generates a $Y$-dependent lower limit of integration, $P_{\min }$. The expression for $P_{\|}$was obtained by energy-momentum conservation at the nuclear vertex in Fig 2, disregarding the recoil energy of the $A-1$ nuclear system.

We conclude this subsection by noting that in the forward limit, $\Delta \rightarrow 0$, Eqs.(29) 36) correctly reproduce the nuclear formulae obtained e.g. in Refs. [25, 38].

\section{Numerical Results}

We calculated $H^{A}$ in both the on-shell and off-shell cases using the nuclear model from Ref. [38] and a parametrization for $H^{N}$ at $\zeta=0$ from Ref. [6]. All results are presented for the ${ }^{4} \mathrm{He}$ nucleus, although our formalism applies more generally to complex nuclei with larger A. ${ }^{8}$

\section{Off-forward EMC Effect}

In Fig 4 we show the $H^{N(A)}(X, 0, t)$ plotted vs. $X$, for a nucleus $\left({ }^{4} H e\right)$ and for the proton, both normalized to 1 , and for $t=0$ and $t=0.1 \mathrm{GeV}^{2}$, respectively. Because ${ }^{4}$ He has a much larger drop with $t$ due to the nuclear form factor behavior, we consider the ratio:

$$
R^{A}(X, \zeta=0, t)=\frac{H^{A}(X, t) / F^{A}(t)}{H^{N}(X, t) / F^{N}(t)}
$$

where: $H^{N(A)}(X, t) \equiv H^{N(A)}(X, \zeta=0, t)$, and $F^{N}$ is the Dirac form factor for a proton. $R^{A}$ becomes equal to the EMC ratio in the forward limit $t=0$. The following normalizations hold:

$$
\int d X \frac{H^{N(A)}(X, t)}{F^{N(A)}(t)}=1 .
$$

By choosing the form for $R^{A}$ in Eq.(39), one eliminates the, somewhat spurious, $t$ dependence coming from the mere comparison of form factors of nuclei with different $A$ (see Fig (4). Nevertheless, $R^{A}$ includes contributions from both the long range and short range nuclear structure. In Fig 5 we show $R^{A}$ as a function of $X$ for different values of $t\left(t=0 \mathrm{GeV}^{2}\right.$, and $t=0.5 \mathrm{GeV}^{2}$ ), both including (Eq.(29)), and disregarding (Eq.(136) ) off-shell effects. Notice that the curves for $t=0$ do not coincide exactly with the "EMC"-like ones in Fig 3 ,

\footnotetext{
${ }^{8}$ Calculations for other complex nuclei are available under request at the e-mail addresses listed here.
} 
because they are divided by the proton, while Fig 3 includes nuclear effects in the deuteron target. The EMC-effect is both enhanced in magnitude, and shifted to lower values of $X$, at $t \neq 0$. Furthermore the effects of dynamical off-shellness are enhanced. As we explain also below, it should be clarified that such an enhancement is not a direct consequence of having probed through GPDs partonic and/or nucleonic transverse degrees of freedom. In fact, because $\Delta$ and the targets intrinsic transverse momenta are decoupled, the $t$ dependence in $R^{A}$ originates from the modification of the longitudinal variable: $X \rightarrow X /\langle Y(A, t)\rangle$, the average value of $Y$ being calculated using Eq.(26).

In Fig 6, we show the $X$ behavior of $R^{A}$ for different values of $t$, in separate panels: longitudinal convolution (left), off-shell effects (right). The marked difference at $x=0.1$ was unexpected according to estimates based on the longitudinal convolution [27]. The effect predicted in this paper is quite sizable and it should be measurable within the accuracy of data currently been analyzed at HERMES.

\section{2. $\quad$-dependence and Role of Long Range Nuclear Interactions}

In order to proceed further, one must devise a method to disentangle the different contributions from both the short and long range nuclear effects originating from the $t$ dependent function $\rho_{A}$ in Eq.(29). A number of long range effects that are well known since the early evaluations of nuclear form factors, are also present in principle for the nuclear GPDs. In a nutshell, one must include terms beyond the nuclear impulse approximation - the so-called two-body and three-body current contributions from the coupling of the virtual photon with a mesonic component in the nucleus, and with a nucleon resonance, respectively [27].

To what extent are these terms affecting the ratio $R^{A}$ ?

In order to evaluate their separate contributions, we consider a Taylor expansion of Eq. (39) around the value $Y=1$, and $P^{2}=M^{2}$ :

$$
\begin{aligned}
R^{A}(X, t) & \approx 1+\frac{\left\langle Y_{1}^{A}(t)\right\rangle}{F^{A, p o i n t}(t)} \times\left[\frac{1}{H^{N}(X)} \frac{\partial H^{N}(X, t)}{\partial X} X\right] \\
& +\frac{\left\langle Y_{2}^{A}(t)\right\rangle}{F^{A, p o i n t}(t)} \times\left[X^{2} \frac{\frac{\partial^{2} H^{N}(X, t)}{\partial X^{2}}-2 X \frac{\partial H^{N}(X, t)}{\partial X}}{2 H^{N}(X)}\right] \ldots
\end{aligned}
$$

where, from Eqs. (36) and (48), $F^{A}(t)=F^{A, p o i n t}(t) F^{N}(t)$, with:

$$
\begin{aligned}
F^{A, \text { point }}(t) & =\int_{0}^{A} d Y \rho_{A}(Y, t) \\
\left\langle Y_{1}(A, t)\right\rangle & =\int_{0}^{A} d Y \rho_{A}(Y, t)(1-Y) \\
\left\langle Y_{2}(A, t)\right\rangle & =\int_{0}^{A} d Y \rho_{A}(Y, t)(1-Y)^{2},
\end{aligned}
$$

$F^{A, p o i n t}$ is the nuclear form factor, calculated by assuming point-like nucleon structure. $Y_{i}$, $i=1,2$ are proportional to higher nuclear moments. Eq.41] 4 helps us understand the behavior in $X, A$, and $t$ of $R^{A}$, from the observation that such ratio is determined by a combination 
of the values of $\left\langle Y_{1(2)}^{A}(t)\right\rangle \approx\left\langle Y_{1(2)}(A, 0)\right\rangle$, and the slope of $H^{N}$ vs. $X$. This can be seen more intuitively by taking a cruder approximation for the numerator of $R^{A}$ :

$$
\frac{H^{A}(X, t)}{F^{A}(t)} \approx \frac{1}{F^{N}(t)} H^{N}\left(\frac{X}{\left\langle Y^{\text {norm }}(A, t)\right\rangle}, t\right),
$$

where $Y^{\text {norm }}(A, t)$ is the $t$-dependent average nucleon momentum in a nucleus, normalized to unity. The extent to which $H^{A}$ on the 1.h.s differs from the free nucleon case is governed by the rescaling in $X$ shown on the r.h.s.. This, in turn, depends both on the size of the deviation from unity of the nucleon momentum $\left\langle Y^{\text {norm }}\right\rangle$, and on the steepness of the slope of $H^{N}$ in $X$.

In the forward limit, $Y_{1}(A, t=0)=\bar{E}_{A} / M-(1 / 3)\left\langle P^{2}\right\rangle_{A} / M^{2}, Y_{2}(A, t=0)=$ $(1 / 3)\left\langle P^{2}\right\rangle_{A} / M^{2}$ [38]. Notice that all the higher order terms in the series have a similar structure, namely they are products of a $\left\langle Y_{n}(A, t)\right\rangle, n>1$ term, and a $n^{\text {th }}$ order derivative of $H^{N}$ divided by $H^{N}$. The terms: $\left\langle Y_{1(2)}(A, t)\right\rangle / F^{A, p o i n t}(t)$, and $\left(X \partial H^{N}(X, t) / \partial X\right) / H^{N}(X, t)$, $\left(X^{2} \partial^{2} H^{N}(X, t) / \partial^{2} X^{2}\right) / H^{N}(X, t)$ are plotted in Fig[7 vs. $t$, from which we see that the $t$ dependence coming from the $\left\langle Y_{1(2)}(A, t)\right\rangle$ terms is relatively very small.

The $t$ dependence of $R^{A}$ shown Figs 5 and 6 can be understood, therefore within the nuclear impulse approximation. The mechanism that produces the modifications of the bound nucleon GPD, and the predicted increase of the effect at $t \neq 0$, is, similarly to the forward case, a reduction of the quarks momentum inside the nuclear medium. Such reduction as we will show in more detail in Section IV is enhanced at $t \neq 0$. As a final remark, we have explained the off-forward EMC effect using the longitudinal convolution approach while being however aware (see Fig 33) that such an approach does not either include the correct physics, or predict the magnitude of the effect. The convolution approach served us however as a guidance. One can draw similar conclusions, in fact, by considering the case $P^{2} \neq M^{2}$, where one an extra term dependent on nucleon off-shellness can be added to the expansion in Eq.(41). This term has the same structure with respect to its $t$-dependence as the other terms in the "on-shell" series. In other words, nucleon off-shellness further depletes the bound nucleon's momentum.

An important conclusion of our study is, therefore, that details of the long range nuclear interactions seem to have little influence on the bound nucleon GPDs.

\section{3. $t$-dependence as a Constraint on GPD Parametrizations}

In Fig 8 we show the $t$ dependence of the ratio $R^{A}$, for both the on-shell (left panel) and off-shell (right panel) cases, for three different values of $X, X=0.1,0.3,0.6$. One can observe markedly different behaviors in the slopes in the two panels. At $X=0.6$ and $t>0.1 \mathrm{GeV}^{2}$ one can observe a decreasing slope both in the on-shell and off-shell cases. This characteristic behavior provides a method to use nuclear effects to distinguish between different parametrizations of GPDs. Recently, a number of new models were proposed that improve on factorized ansatze of the type $H(X, \zeta, t)=H(X, \zeta) F(t)$, used in many earlier theoretical evaluations. The models are obtained for $\xi(\zeta=0)$. include parametrizations based on an exponential fall-off with $t$ of the type [4, 43, 44]:

$$
H(x, t)=f(x) \exp [a(x) t]
$$

By inspecting Eq.(39), one can conclude that: 
i) No $t$ dependence of $R^{A}$ should be observed in a factorized model;

ii) The exponential models predict a linear dependence in $t$ originating from the $X$ derivative of $H^{N}$;

iii) Other models predict a $t$ dependence that deviates from the linear behavior. The comparison of exponential type parametrizations and the quark-diquark model used in this paper is presented in Fig 9.

\section{SUM RULES IN NUCLEI}

Mellin moments of order $n$ for the nucleon GPDs can be defined, analogously to the forward case, by integrating over $x$ the matrix elements in Eqs.(9) and (16), multiplied by $x^{n}$. ${ }^{9}$ Differenty from the forward case, the moments will depend on the variables $t$ and $\xi$, besides displaying the expected $Q^{2}$ dependence. In other words, they can be written as $t$-dependent form factors of the same matrix elements of twist two local operators that appear in the forward case, $(t=0)$. Special cases are given by the form factors $(n=0)$ :

$$
\begin{aligned}
\int_{-1}^{1} d x H_{q}(x, \xi, t) & =F_{1}^{q}(t) \\
\int_{-1}^{1} d x E_{q}(x, \xi, t) & =F_{2}^{q}(t),
\end{aligned}
$$

where $q=u, d, s, \ldots$, and by the second moments $(n=1)$, expressed through the symmetric energy-momentum tensor, in terms of the form factors of the quarks momentum, $M_{2}^{q}(t)$, and angular momentum, $J^{q}(t)$, respectively [2]:

$$
\begin{aligned}
& \int_{-1}^{1} d x x H_{q}(x, \xi, t)=M_{2}^{q}(t)+\frac{4}{5} d_{1}(t) \xi^{2} \\
& \int_{-1}^{1} d x x E_{q}(x, \xi, t)=\left(2 J^{q}(t)-M_{2}^{q}(t)\right)-\frac{4}{5} d_{1}(t) \xi^{2}
\end{aligned}
$$

$M_{2}^{q}(0)$ is the total momentum fraction carried by the quarks, $J^{q}(0)$ is the total angular momentum. $d_{1}(t)$ is the moment of the first gegenbauer coefficient in the expansion of the $D$-term (see 10] and references therein). Also, we do not write explicitly the dependence on $Q^{2}$, unless necessary. ${ }^{10}$ Similar expressions exist for the gluon components.

The sum rules in a nucleus can be constructed from Eqs. (48) and (50) as:

$$
\begin{aligned}
\int_{-A}^{A} d x H^{A}(x, \xi, t) & =F^{A}(t) \\
\int_{-A}^{A} d x x H^{A}(x, \xi, t) & =M_{2}^{A}(t)+\frac{4}{5} d_{1}^{A}(t) \xi^{2}
\end{aligned}
$$

In [41] the connection was shown between $d_{1}^{A}(0)$ and the components of the energymomentum tensor describing the shear forces in the hadronic system. We can evaluate

\footnotetext{
${ }^{9}$ We use the notation in Eq.(10) that is more appropriate for the discussion of Mellin moments.

10 The original derivation of Eq.(50) was in fact what motivated the introduction of GPDs in Refs. 2, 3].
} 
the Mellin moments in a nucleus according to the microscopic approach described in the previous Sections. By restricting to the valence quarks contributions only $(x, y>0)$, and by disregarding off-shell effects, one has the intuitive factorized form:

$$
M_{n}^{A}(t)=\left(\int_{0}^{A} d y y^{n-1} f_{A}(y, t)\right)\left(\int_{0}^{1} d x x^{n-2}\left[x H^{N}(x, \xi, t)\right]\right),
$$

where $f_{A}(y, \xi, t) \approx f_{A}(y, \xi=0, t) \equiv f_{A}(y, t)$ in a nucleus, within a non-relativistic description. For $n=1$ and $n=2$ one has specifically:

$$
\begin{aligned}
F^{A}(t) & =F^{A, \text { point }}(t) F^{N}(t) \\
M_{2}^{A}(\xi, t) & =M_{2}^{A, \text { point }}(t) M_{2}^{N}(t)+M_{0}^{A, \text { point }}(t) \frac{4}{5} d_{1}^{N}(t) \xi^{2},
\end{aligned}
$$

with $M_{n}^{A, \text { point }}(t)=\int d y y^{n-1} f_{A}(y, t)$, the nuclear moment obtained by considering "pointlike" nucleons. At $\xi=0$ one has:

$$
M_{2}^{A}(t)=M_{2}^{A, p o i n t}(t) M_{2}^{N}(t)
$$

related to the average value of the longitudinal momentum carried by the quarks in a nucleus:

$$
\langle x(t)\rangle_{A}=\frac{M_{2}^{A}(t)}{F^{A}(t)}=\frac{M_{2}^{A, \text { point }}(t)}{F^{A, p o i n t}(t)} \frac{M_{2}^{N}(t)}{F^{N}(t)}=\langle y(t)\rangle_{A}\langle x(t)\rangle_{N},
$$

The D-term in a nucleus reads:

$$
d_{1}^{A}(t)=M_{0}^{A, p o i n t}(t) d_{1}^{N}(t)
$$

At $t=0$ one has: $d_{1}^{A}(0) \approx 1 /\left[1-\bar{E} / M+2 / 3\left\langle P^{2}\right\rangle / 2 M^{2}\right]_{A} d_{1}^{N}(0)$. The microscopic calculation presented here predicts therefore an $A$-dependence of $d_{1}^{A}(0) \propto A$, modulo a $\propto \ln A$ enhancement from nuclear dynamics, i.e. from the $A$-dependent term multiplying $d_{1}^{N}(0)$. This is at variance with the estimate, $d_{1}^{A}(0) \propto A^{7 / 3}$ obtained by calculating directly the nuclear energymomentum tensor in a liquid drop picture of the nucleus [41]. Whether the two pictures can be reconciliated, that is addressing the question of whether the shear forces are either under- or overestimated in either model, is an interesting problem for future investigations.

Finally, we discuss how the moments of GPDs in nuclei might provide new insight on the origin of nuclear medium modifications of hadrons. Two distinct pictures based on hadronic and partonic degrees of freedom respectively have been put forth to explain the effect. On one side, the convolution formalism discussed here makes use of "hadronic" degrees of freedom, although the hadronic structure can be modified due to off-shell effects. The nature of such modifications resides in the parameters of nucleon dynamics and interactions. On the other hand, other descriptions (16] and references therein), relate the modifications of "partonic" parameters such as the string tension or the confinement radius to density dependent effects in the nuclear medium. The authors of Ref. 17] (CRR) in fact, even formulated the hypothesis of a "duality" scenario between the two pictures. A qualitatively different description that would help disentangle the two, can be obtained from the investigation of form factor (or spatial) type quantities such as the ones described in Eqs. (48) 58). The effect of partial deconfinement predicts a similar formula for $M_{2}^{A}\left(t, Q^{2}\right), \xi=0$ calculated in the convolution formalism, namely:

$$
M_{2}^{A, C R R}\left(t, Q^{2}\right)=\left[\left(1-d_{N S}^{n=2} \kappa_{A}\right) F^{A, p o i n t}(t)\right] M_{2}^{N}\left(t, Q^{2}\right),
$$


$d_{N S}^{n=2}$ being the anomalous dimensions for the Non-Singlet $(N S)$ sector, and $\kappa_{A}$ being the parameter modifying the $Q^{2}$ scale dependence in Ref. 17]. In Fig[10] we show the ratio of $M_{2}^{A} / F^{A}$ over $M_{2}^{N} / F^{N}$ in several scenarios: the longitudinal convolution formula where the ratio coincides with $\langle y(t)\rangle_{A}$ from Eq. (57); the ratio calculated including off-shell effects (that break the factorization in Eq.(57)); and the prediction from Eq.(59). The most important aspect of this graph is that while "factorization based" approaches such as the longitudinal convolution formalism and the CRR-model, predict a $t$ dependence of the ratio that is approximately flat, off-shell effects have an impact on the $t$ dependence, and are therefore indispensable for treating the transverse spatial dependence (or $3 D$ imaging) in a nucleus, a subject to be explored in future studies [37.

Besides the results on the $N S$ contribution, yet another interesting possibility emerges from the non-forward scattering extension of nuclear deep inelastic structure studies. Predictions were given in Ref. [17] on the behavior of the binding quanta appearing in the expression for the second moment for the sea quarks. A relation was found between the sea quarks, $q_{s}$ component of the "binding quanta", $B, M_{2}^{q_{s} / B}$, and the gluon component of the nucleon $M_{2}^{G / N}$ :

$$
M_{2}^{q_{s} / B}\left(Q^{2}\right) \approx-\frac{3}{16} f M_{2}^{G / N}\left(Q^{2}\right)
$$

from which it was deduced that the binding quanta, identified in [17] with nuclear pions, are gluonic in nature, or in other words, they contain gluons that cannot be generated by evolution. GPDs provide a new method to study the form factor of such gluon dominated particles, that could be identified as candidates for glueballs. One can in fact use the perturbative QCD (PQCD) equations of Ref. [17] in conjunction with the formalism described above to obtain:

$$
M_{2}^{q_{s} / B}(t)=\frac{1}{M_{2}^{B / A}(t)}\left(M_{2}^{q_{s} / A}(t)-M_{2}^{q_{s} / N}(t) M_{2}^{N / A}(t)\right) .
$$

\section{CONCLUSIONS}

We conducted an exploratory study using on one side GPDs as tools to unravel the deep inelastic transverse structure of nuclei, and on the other obtaining information on GPDs themselves by observing how they become modified in the nuclear environment. We used a microscopic approach restricted mainly to the valence structure. We conclude that:

i) Although GPDs probe transverse degrees of freedom in a nucleus, through the variable $t$, modifications of the transverse momentum dependence are described similarly to the forward case, through the $k_{\perp}, P_{\perp}$ variables. These variables are however not Fourier conjugates to $\Delta$, thus rendering the description of off-shell effects more subtle.

ii) The unraveled $t$ dependence of nuclear modifications originates from different mechanisms at the nuclear and nucleon vertices, respectively. By taking nuclear ratios of GPDs normalized to form factors, we demonstrated that the details of the nuclear long range interactions (two-body currents, large distance behavior of nuclear density, etc. ...) can be disregarded compared to the deep inelastic induced modifications of the bound GPDs. This result bears important consequences since it allows us on one side to avoid the intricacies and details related to the evaluation of nuclear form factors, and on the other, it points at interesting physical phenomena determined by the short range part of the nuclear interactions. 
iii) The pattern of nuclear modifications predicted, and in particular the deviations of offshell effects from the longitudinal convolution provide clear signals to be sought in experimental measurements.

iv) Data on DVCS in nuclei will help one distinguish among models for GPDs. Distinctive behaviors in e.g. the $t$ dependence emerge for the factorized, the exponential based, and other LC based models presented here.

v) Interesting relationships were found by studying Mellin moments in nuclei. In particular, we predicted the $A$-dependence for the $D$-term of GPDs within a microscopic approach, and the behavior with $t$ of the total momentum carried by quarks in a nucleus. By studying Mellin moments we were able to make a connection with widely used approaches that relate the modifications of "partonic" parameters such as the string tension or the confinement radius to density dependent effects in the nuclear medium [16, 17]. We consider this an important result of our paper at the light of nuclear hadronization studies which are vital for interpreting current and future data at RHIC, HERMES and Jefferson Lab ([16] and references therein).

Many questions remain, some of which will be addressed in a forthcoming paper [37. These will include a detailed study of the impact parameter $(b)$ dependence, and predictions of quantities measurable at currently available energies, namely both beam and target asymmetries, including a quantitative evaluation of the coherent vs. incoherent nuclear contributions.

\section{Acknowledgments}

We thank Delia Hasch for discussing HERMES experiments, and Wally Melnitchouk for discussions. This work is supported by the U.S. Department of Energy grant no. DE-FG0201ER41200.

\section{APPENDIX: OFF-FORWARD PROPAGATOR}

We evaluate the trace in Eq.(18), that gives origin to the square root factors in our model for nucleon GPDs, Eq.(21).

We start from defining the sum over the spins in Eq.(18), for particles with different momenta, $k \equiv(E ; \mathbf{k})$, and $k^{\prime}=k+\Delta$. The spinors are defined as:

$$
u(k, s)=N(\not k+m) u(0, s),
$$

with: $u_{1}(0, s)=1, u_{i}(0, s)=0, i=2,3,4,\left(u_{2}(0,-s)=1, u_{i}(0,-s)=0, i=1,3,4\right)$; and $N^{-1}=(E+m)^{1 / 2}$. In the forward case, therefore:

$$
\sum_{s} u(k, s) \bar{u}(k, s)=(\not k+m)
$$

In the off-forward case:

$$
\begin{aligned}
\sum_{s} u(k, s) \bar{u}\left(k^{\prime}, s\right) & =N N^{\prime} \sum_{s}(\not k+m) u(0, s) \bar{u}(0, s)\left(\not k^{\prime}+m\right) \\
& =N N^{\prime}(\not k+m) \frac{\left(1+\gamma_{o}\right)}{2}\left(\not k^{\prime}+m\right)
\end{aligned}
$$


The trace of this quantity is given by:

$$
\operatorname{Tr}\left\{\sum_{s} u(k, s) \bar{u}\left(k^{\prime}, s\right)\right\}=2 N N^{\prime}\left[\left(k . k^{\prime}+m^{2}\right)+m\left(k_{0}+k_{0}^{\prime}\right)\right]
$$

In light cone coordinates the above is expressed as:

$$
\begin{aligned}
\operatorname{Tr}\left\{\sum_{s} u(k, s) \bar{u}\left(k^{\prime}, s\right)\right\}= & \frac{2}{\sqrt{\left(\frac{k^{+}+k^{-}}{\sqrt{2}}+m\right)\left(\frac{k^{\prime+}+k^{\prime-}}{\sqrt{2}}+m\right)}}\left[\left(k^{+} k^{\prime-}+k^{-} k^{\prime+}-k_{\perp} k_{\perp}^{\prime}+m^{2}\right)\right. \\
& \left.+\frac{m}{\sqrt{2}}\left(\left(k^{+}+k^{-}\right)+\left(k^{\prime+}+k^{\prime-}\right)\right)\right]
\end{aligned}
$$

thus, at leading order:

$$
\operatorname{Tr}\left\{\sum_{s} u(k, s) \bar{u}\left(k^{\prime}, s\right)\right\}=2 m \frac{\left(k^{+}+k^{\prime+}\right)}{\sqrt{k^{+} k^{\prime+}}}=2 m \frac{2 X-\zeta}{\sqrt{X(X-\zeta)}}
$$

and the trace with $\gamma^{+}$appearing in Eq.(21), is given by:

$$
\frac{1}{2 P^{+}} \operatorname{Tr}\left\{\gamma^{+} \sum_{s} u(k, s) \bar{u}\left(k^{\prime}, s\right)\right\}=\frac{2}{P^{+}} \sqrt{k^{+} k^{\prime+}}=2 \sqrt{X} \sqrt{X-\zeta}
$$

[1] D. Muller, D. Robaschik, B. Geyer, F. M. Dittes and J. Horejsi, Fortsch. Phys. 42, 101 (1994)

[2] X. D. Ji, Phys. Rev. D 55, 7114 (1997)

[3] A. V. Radyushkin, Phys. Rev. D 56, 5524 (1997)

[4] M. Burkardt, Int. J. Mod. Phys. A 18, 173 (2003); ibid Phys. Rev. D 62, 071503 (2000) [Erratum-ibid. D 66, 119903 (2002)].

[5] M. Diehl, Eur. Phys. J. C 25, 223 (2002) [Erratum-ibid. C 31, 277 (2003)]

[6] S. Liuti and S.K. Taneja, Phys. Rev. D 70, 074019 (2004).

[7] M. Diehl, T. Feldmann, R. Jakob and P. Kroll, Eur. Phys. J. C 39, 1 (2005).

[8] D. Chakrabarti and A. Mukherjee, Phys. Rev. D 71, 014038 (2005).

[9] A. V. Belitsky, X. d. Ji and F. Yuan, Phys. Rev. D 69, 074014 (2004).

[10] M. Diehl, Phys. Rept. 388, 41 (2003).

[11] F. Ellinghaus, R. Shanidze, and J. Volmer, hep-ex/0212019 D. Hasch, private communication.

[12] J. P. Ralston and B. Pire, Phys. Rev. D 66, 111501 (2002).

[13] M. Burkardt and G. A. Miller, arXiv:hep-ph/0312190.

[14] J. J. Aubert et al. [European Muon Collaboration], Phys. Lett. B 123, 275 (1983).

[15] D. F. Geesaman, K. Saito and A. W. Thomas, Ann. Rev. Nucl. Part. Sci. 45, 337 (1995).

[16] A. Accardi, D. Grunewald, V. Muccifora and H. J. Pirner, arXiv:hep-ph/0502072.

[17] F. E. Close, R. G. Roberts and G. G. Ross, Nucl. Phys. B 296, 582 (1988).

[18] J. Wambach, Prog. Part. Nucl. Phys. 50, 615 (2003).

[19] G. A. Miller and J. R. Smith, Phys. Rev. C 65, 015211 (2002) [Erratum-ibid. C 66, 049903 (2002)]; ibid Phys. Rev. C 65, 055206 (2002) 
[20] A. W. Thomas, Annalen Phys. 13, 731 (2004); A. W. Thomas, P. A. M. Guichon, D. B. Leinweber and R. D. Young, Prog. Theor. Phys. Suppl. 156, 124 (2004).

[21] S. J. Brodsky, D. S. Hwang and I. Schmidt, Phys. Lett. B 530, 99 (2002).

[22] R.K. Ellis, W. Furmanski and R. Petronzio, Nucl. Phys. B212 29.

[23] S. A. Kulagin, G. Piller and W. Weise, Phys. Rev. C 50, 1154 (1994).

[24] S.L. Adler, Phys. Rev. 1431144.

[25] F. Gross and S. Liuti, Phys. Rev. C 45, 1374 (1992).

[26] E. R. Berger, F. Cano, M. Diehl and B. Pire, Phys. Rev. Lett. 87, 142302 (2001); F. Cano and B. Pire, Eur. Phys. J. A 19, 423 (2004).

[27] A. Kirchner and D. Muller, Eur. Phys. J. C 32, 347 (2003).

[28] A. Freund and M. Strikman, Eur. Phys. J. C 33, 53 (2004).

[29] M. Strikman and C. Weiss, Phys. Rev. D 69, 054012 (2004); L. Frankfurt, M. Strikman and C. Weiss, Phys. Rev. D 69, 114010 (2004).

[30] S. Scopetta, Phys. Rev. C 70, 015205 (2004).

[31] R. Jakob, P. J. Mulders and J. Rodrigues, Nucl. Phys. A 626, 937 (1997), and references therein.

[32] W. Melnitchouk, A. W. Schreiber and A. W. Thomas, Phys. Rev. D 49, 1183 (1994).

[33] K. Goeke, M. V. Polyakov and M. Vanderhaeghen, Prog. Part. Nucl. Phys. 47, 401 (2001).

[34] K. J. Golec-Biernat and A. D. Martin, Phys. Rev. D 59, 014029 (1999).

[35] S. I. Alekhin, S. A. Kulagin and S. Liuti, Phys. Rev. D 69, 114009 (2004)

[36] J. Gomez et al., Phys. Rev. D 49, 4348 (1994).

[37] S. Liuti ans S.K. Taneja, in preparation.

[38] C. Ciofi Degli Atti and S. Liuti, Phys. Lett. B 225 (1989) 215; ibid Phys. Rev. C 41, 1100 (1990).

[39] S. Liuti, in preparation.

[40] J. Osborne and X. N. Wang, Nucl. Phys. A 710, 281 (2002).

[41] M. V. Polyakov, Phys. Lett. B 555, 57 (2003).

[42] C. Ciofi degli Atti and S. Liuti, Phys. Rev. C 44, 1269 (1991).

[43] M. Diehl, T. Feldmann, R. Jakob and P. Kroll, Eur. Phys. J. C 39, 1 (2005).

[44] M. Guidal, M. V. Polyakov, A. V. Radyushkin and M. Vanderhaeghen, arXiv:hep-ph/0410251. 


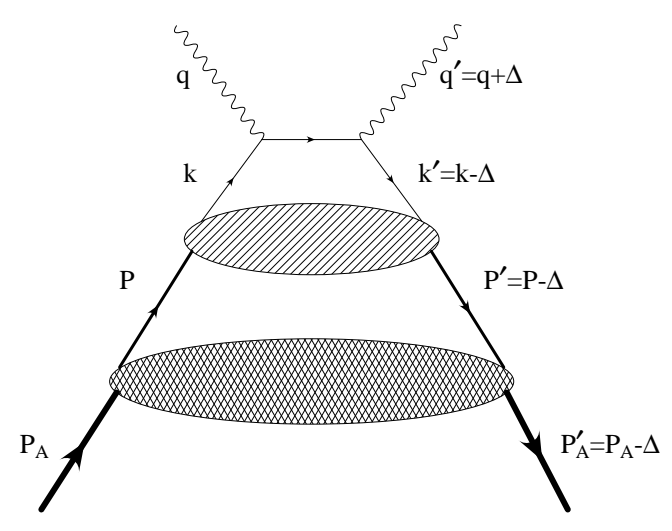

(a)

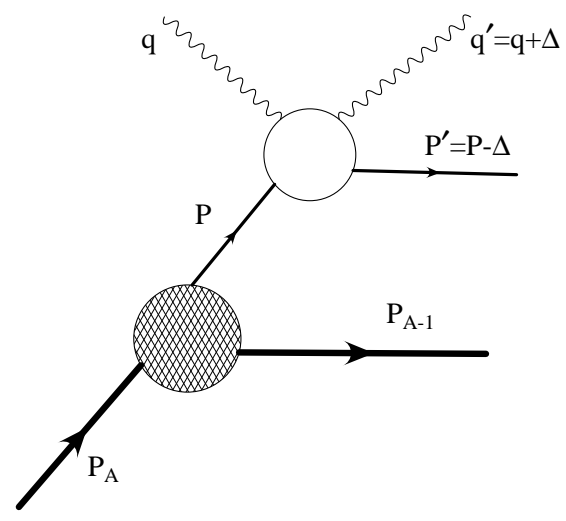

(b)

FIG. 1: Amplitude for DVCS from a nuclear target at leading order in $Q^{2}$. (a) Coeherent process; (b) Incoherent process. For the incoherent process the nucleon handbag diagram is also assumed.

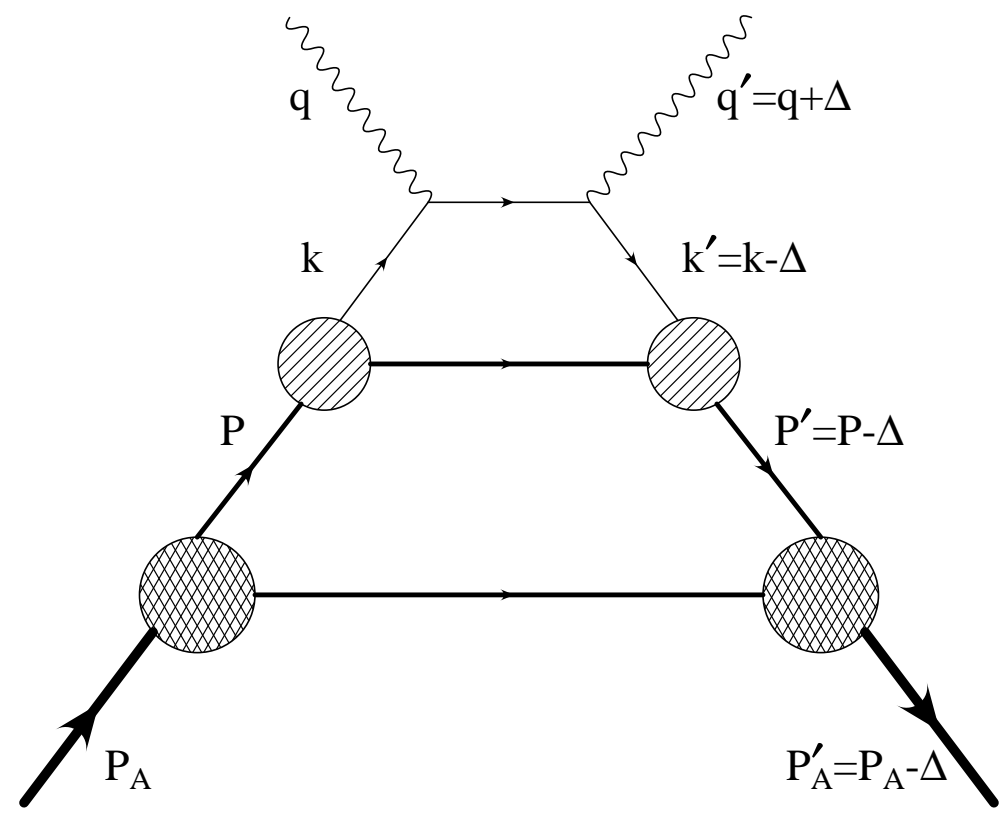

FIG. 2: Off-forward nuclear double convolution diagram. The nucleon is described by a quarkdiquark model, and the nucleus is treated within the Impulse Approximation. 

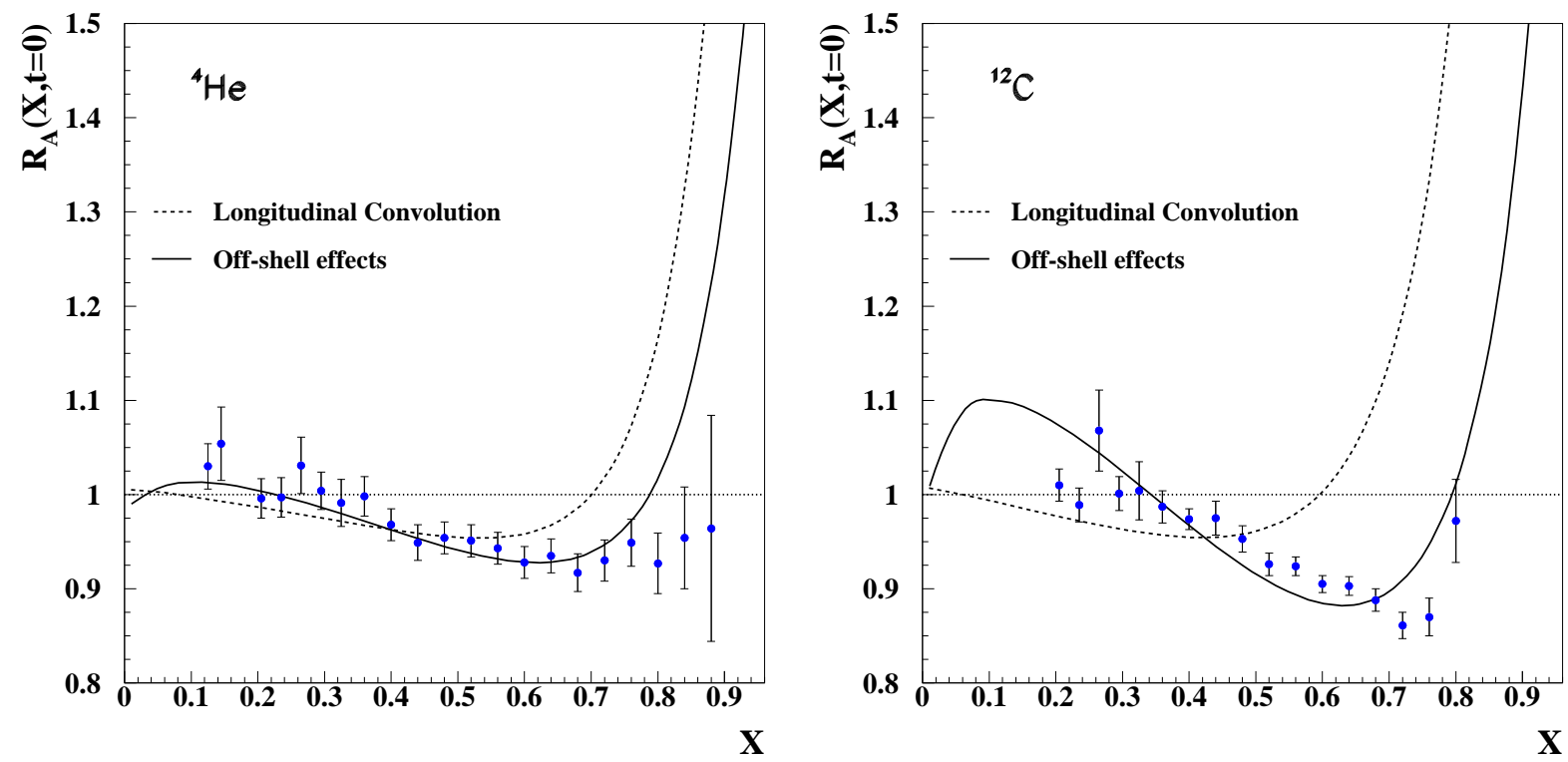

FIG. 3: EMC effect in forward Compton scattering, with off-shell effects (full line), Eq.(29), and calculated with the longitudinal convolution formula, Eq.(36). Left panel: ${ }^{4} \mathrm{He}$, right panel ${ }^{12} \mathrm{C}$. Experimental data from [36]. 


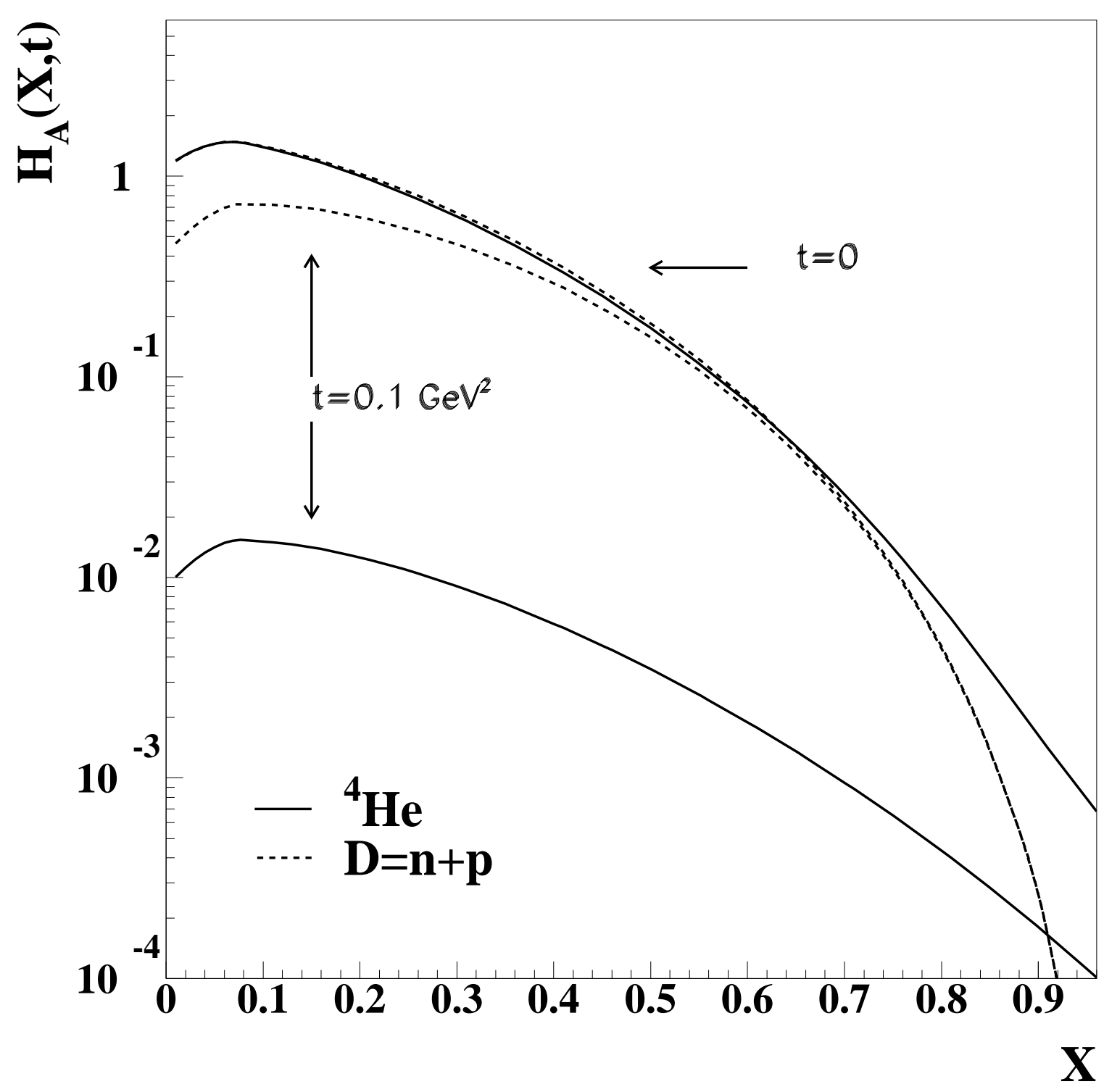

FIG. 4: GPD in ${ }^{4} H e$, divided by the number of nucleons, (full lines) and in the nucleon (dashed lines), evaluated at two different values of $t, t=0$, and $t=0.1 \mathrm{GeV}^{2}$, as respectively indicated by the arrows in the figure. 


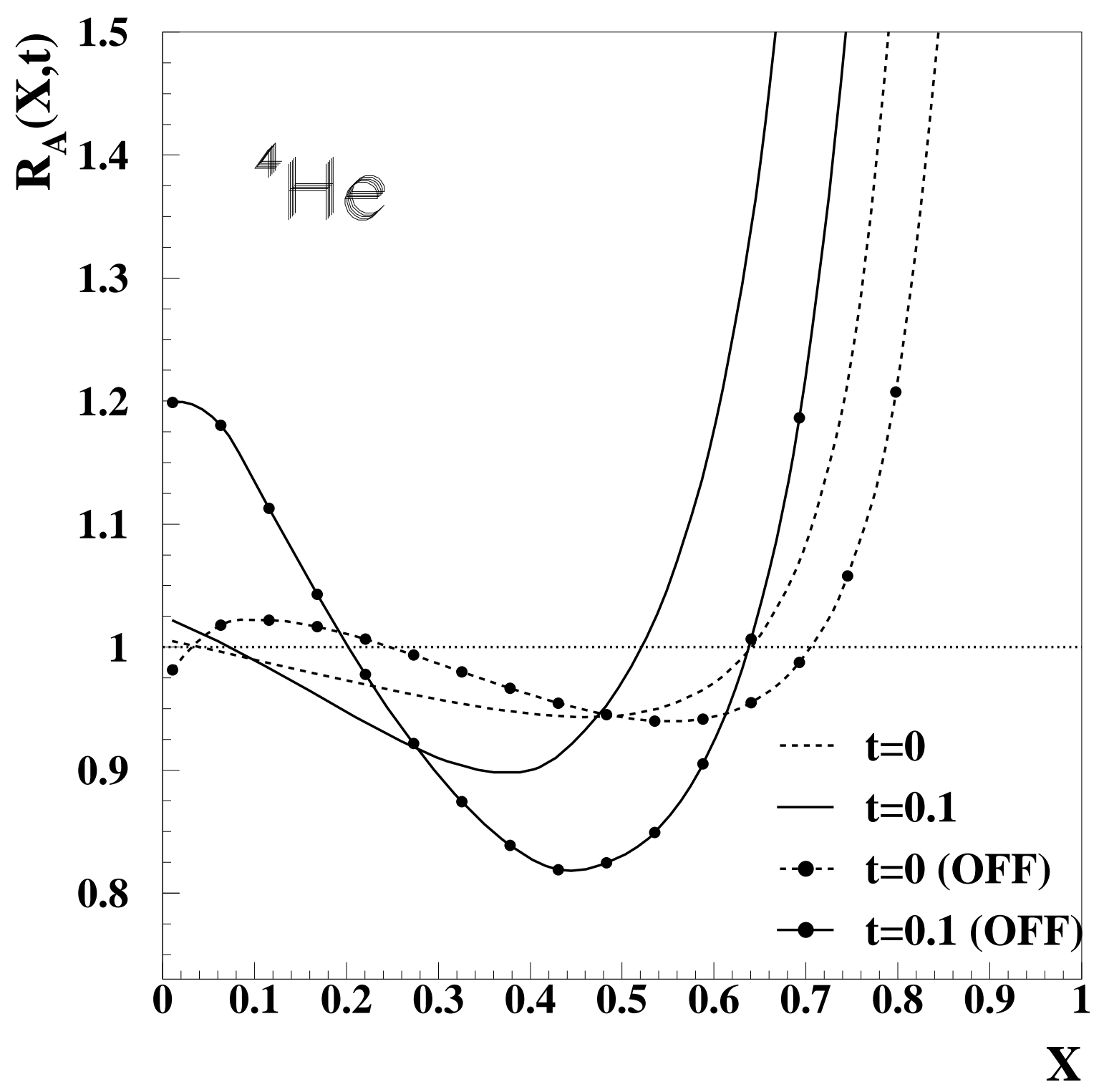

FIG. 5: Off-forward EMC effect in 4 He. The ratio $R^{A}$ represented in the figure is defined in Eq.(39). Full line: including off-shell effects. Dashed line: longitudinal convolution. The full line with bullets includes off-shell effects, at $t=0.1 \mathrm{GeV}^{2}$; the dashed line with bullets was obtained with the longitudinal convolution at $t=0.1 \mathrm{GeV}^{2}$. The lines without bullets correspond to $t=0$. 

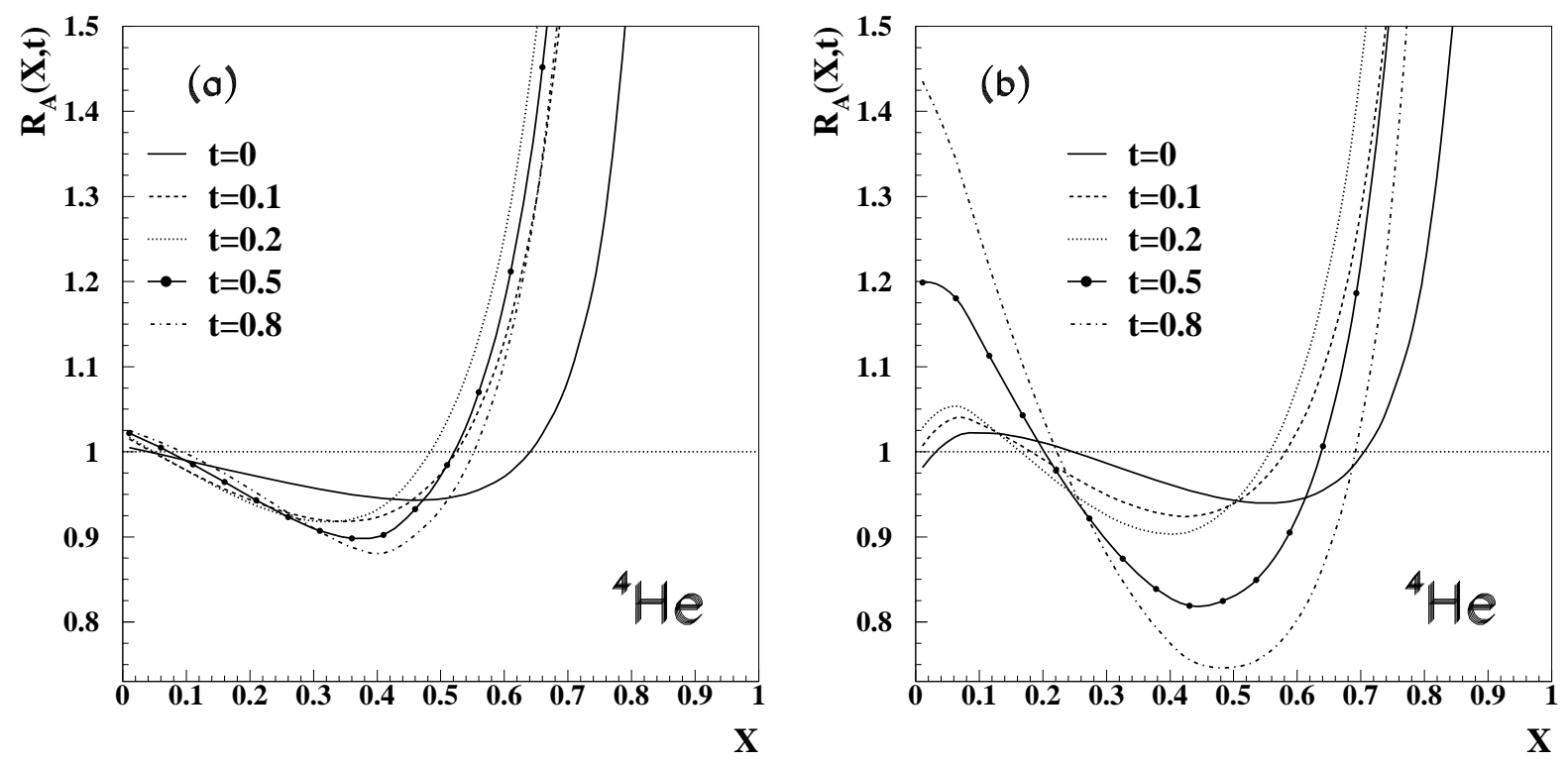

FIG. 6: Comparison of off-forward EMC effect without (panel (a)), and with (panel (b)) off-shell effects. The ratio $R^{A}$ is given in Eq.(39). Several values of $t$ ranging from $t=0$ to $0.8 \mathrm{GeV}^{2}$ are considered, as labeled in the figure. 

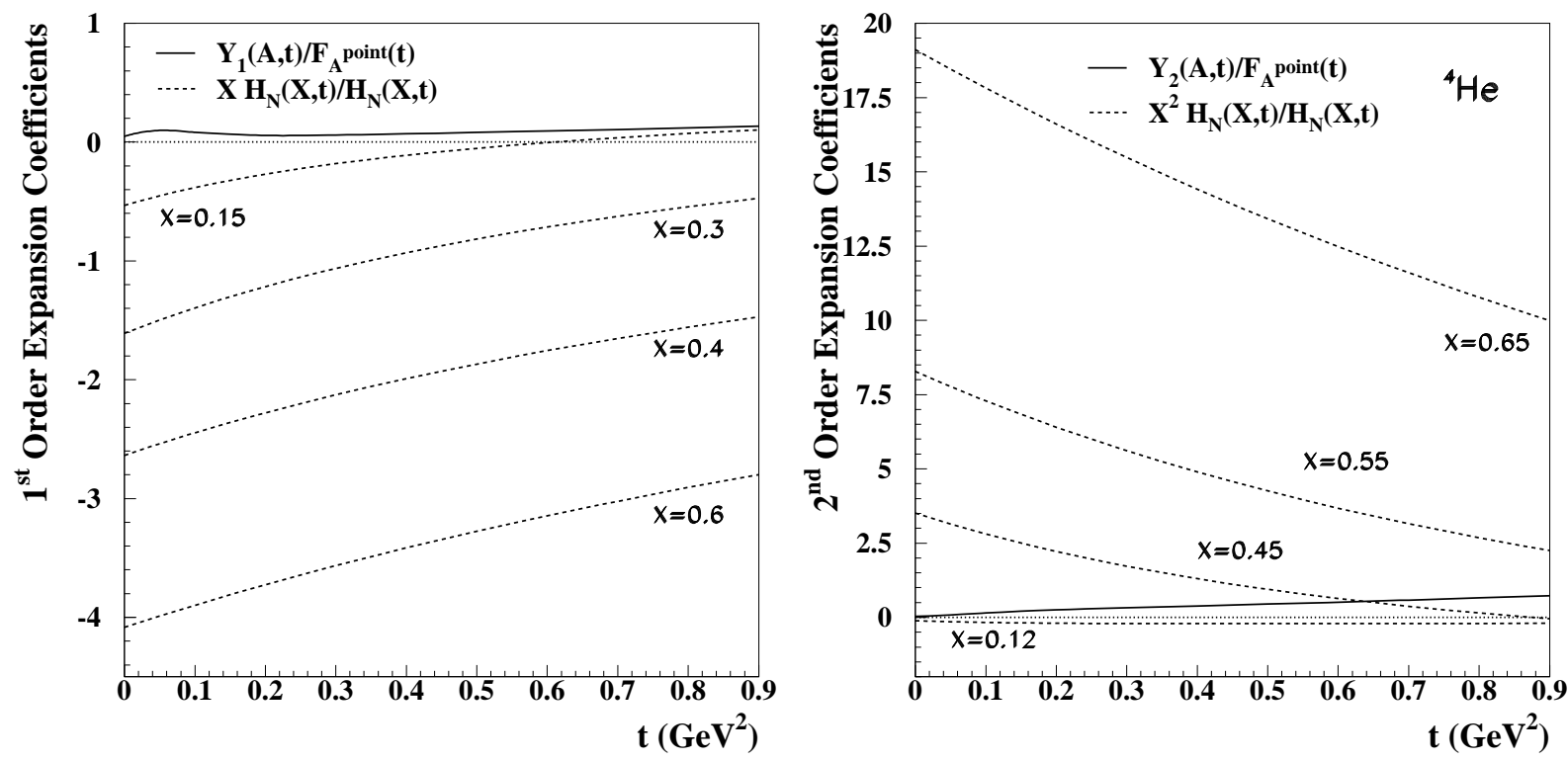

FIG. 7: Comparison between long range nuclear contributions, and deep inelastic type contributions the to the $t$ dependence of the ratio $R^{A}$, Eq. (39). By performing an expansion in the parameter $Y$, Eq. (41), one can pinpoint the driving terms for such dependence. The figure illustrates the first two terms of the expansion, from which the nuclear "form-factor-like" dependence is clearly shown not to affect the ratio. 

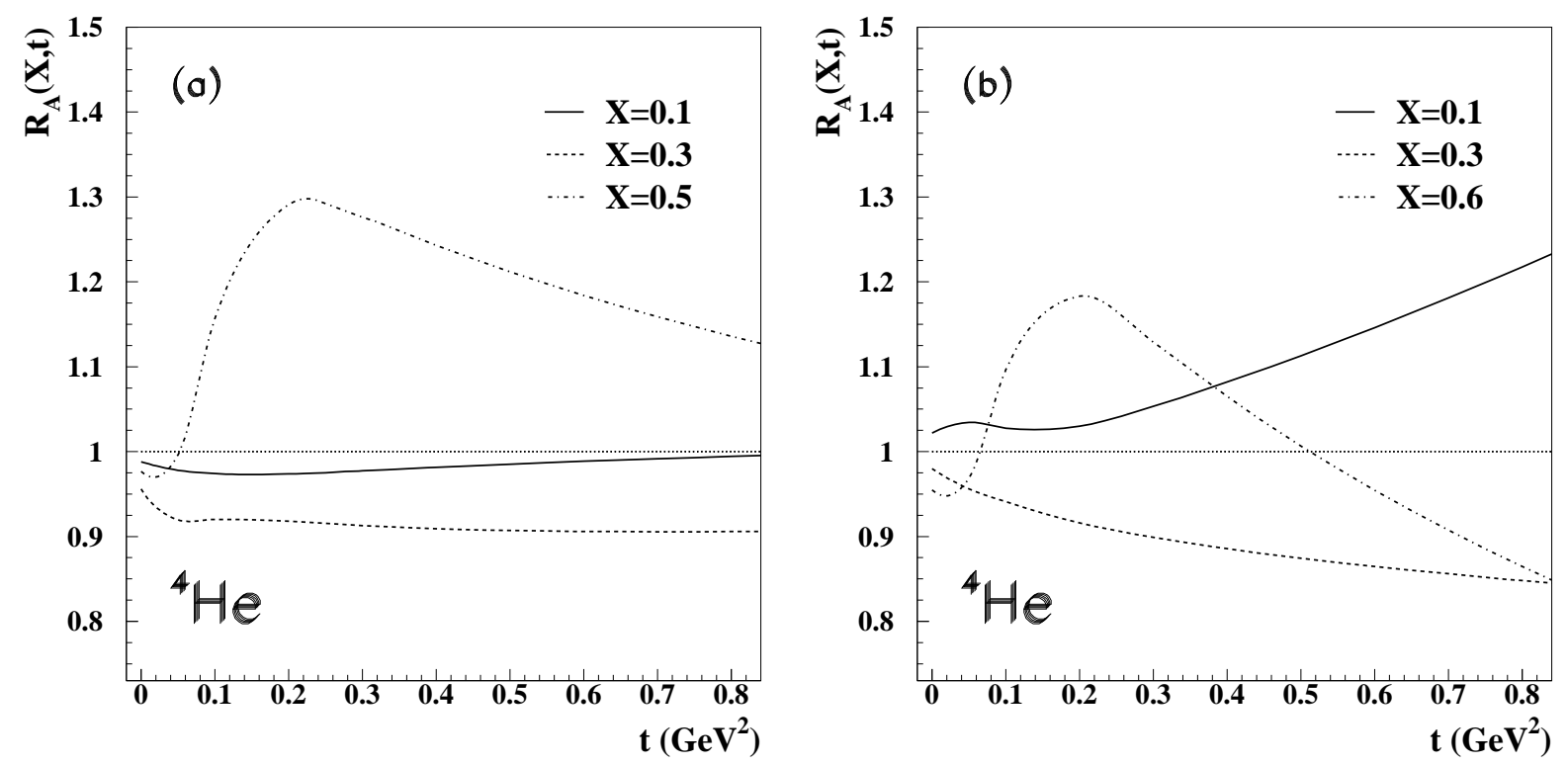

FIG. 8: $t$-dependence of the generalized EMC effect for different values of $X: X=0.1$ (full), $X=0.3$ (short dashes), $X=0.6$ (dot-dashes). (a) No off-shell effects; (b) with off-shell effects. Notice the striking difference between the off-shell and on-shell curves discussed in the text at both low and large values of $X$. 


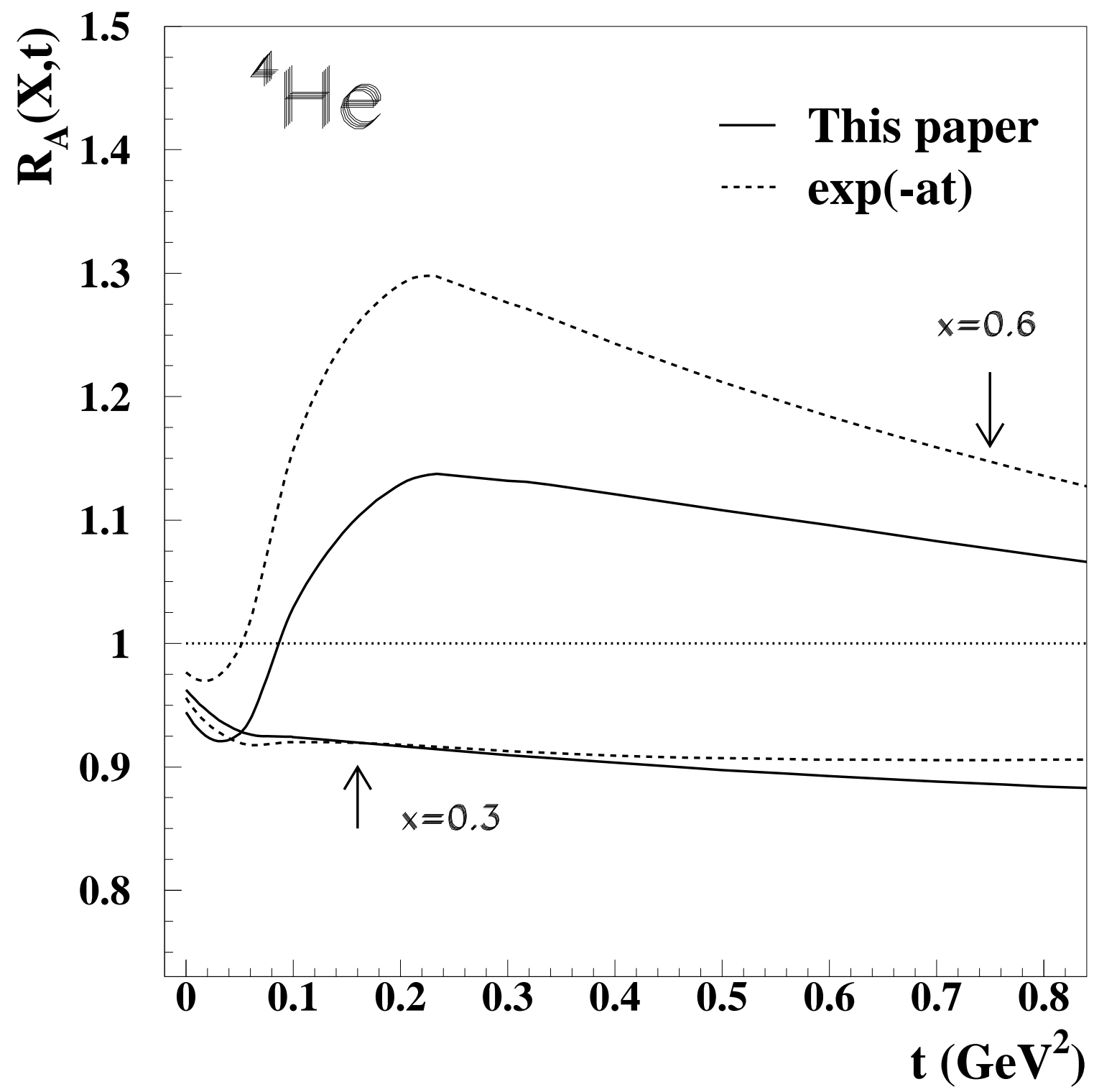

FIG. 9: $t$--dependence of the generalized EMC effect for two different types of parametrizations: The LC wave function one presented in this paper (full curves), and the "exponential" type adopted by several groups $([4,43,44]$ and references therein). 


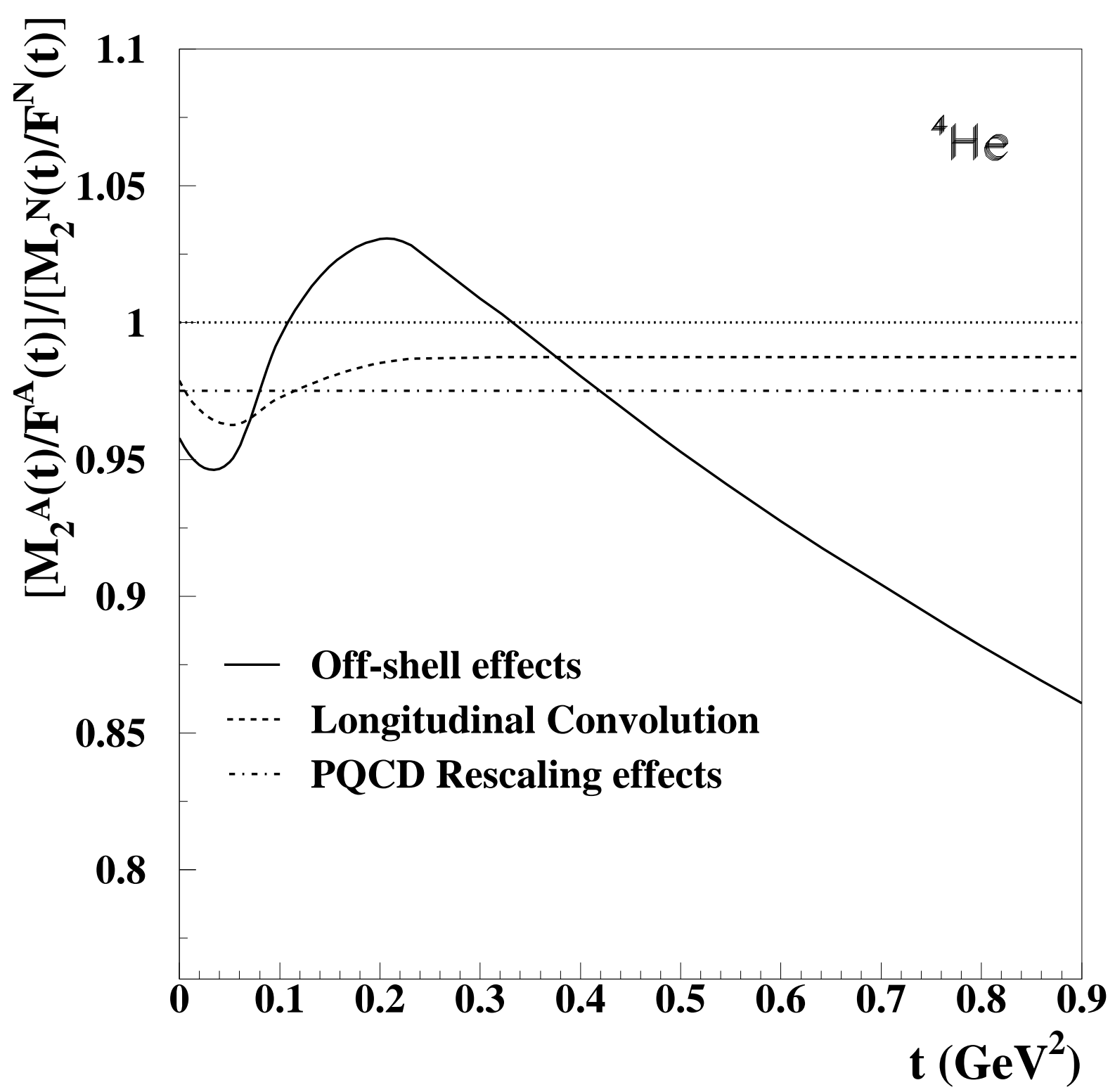

FIG. 10: Ratio of $n=2$ GPD moments of bound nucleons in ${ }^{4} H e$ over the free nucleon ones as a function of $t$. Moments have been normalized to the form factors (see text). The intercept at $t=0$ represents the ratio of the momentum fraction taken by valence quarks in a nucleus with respect to the free nucleon one. 